\title{
Stellar Abundances in the Early Galaxy and Two $r$-Process Components
}

\author{
Y.-Z. Qian ${ }^{1}$ and G. J. Wasserburg ${ }^{2}$
}

Received —

\footnotetext{
${ }^{1}$ T-5, MS B283, Theoretical Division, Los Alamos National Laboratory, Los Alamos, NM 87545; qian@paths.lanl.gov.

${ }^{2}$ The Lunatic Asylum, Division of Geological and Planetary Sciences, California Institute of Technology, Pasadena, CA 91125.
} 


\begin{abstract}
We present quantitative predictions for the abundances of $r$-process elements in stars formed very early in the Galactic history using a phenomenological two-component $r$-process model based on the ${ }^{129} \mathrm{I}$ and ${ }^{182} \mathrm{Hf}$ inventory in the early solar system. This model assumes that a standard mass of the ISM dilutes the debris from an individual supernova. High frequency supernova $\mathrm{H}$ events and low frequency supernova L events are proposed in the model with characteristics determined by the meteoritic data on ${ }^{129} \mathrm{I}$ and ${ }^{182} \mathrm{Hf}$. The yields in an $\mathrm{H}$ or $\mathrm{L}$ event are obtained from these characteristics and the solar $r$-process abundances under the assumption that the yield template for the high mass $(A>130)$ nuclei associated with ${ }^{182} \mathrm{~W}$ or the low mass $(A \leq 130)$ nuclei associated with ${ }^{127} \mathrm{I}$ is the same for both the $\mathrm{H}$ and $\mathrm{L}$ events and follows the corresponding solar $r$-pattern in each mass region. This choice of the yield templates is justified by the regular solar-like $r$-process abundance pattern for $\mathrm{Ba}$ and higher atomic numbers observed in very metal-poor stars.

The abundance of $\mathrm{Eu}$, not $\mathrm{Fe}$, is proposed as a key guide to the age of very metal-poor stars. We predict that stars with $\log \epsilon(\mathrm{Eu})=-2.98$ to -2.22 were formed from an ISM contaminated most likely by a single $\mathrm{H}$ event within the first $\sim 10^{7}$ yr of the Galactic history and should have an $\mathrm{Ag} / \mathrm{Eu}$ abundance ratio less than the corresponding solar $r$-process value by a factor of at least 10. Many of the very metal-poor stars observed so far are considered here to have been formed from an ISM contaminated by many $(\sim 10) r$-process events. Stars formed from an ISM contaminated only by a pure L event would have an $\mathrm{Ag} / \mathrm{Eu}$ ratio higher than the corresponding solar $r$-process value but would be difficult to find due to the low frequency of the L events. However, variations in the relative abundances of the low and high mass regions should be detectable
\end{abstract}


in very metal-poor stars.

Subject headings: Galaxy: evolution - nuclear reactions, nucleosynthesis, supernova, abundances — stars: abundances — stars: Population II 


\section{Introduction}

In this paper, we present the estimated abundances of elements produced by two distinct types of $r$-process events based on a phenomenological model for the production of the relevant nuclei. There are ongoing active investigations determining elemental abundances in very metal-poor stars with particular emphasis on the abundances of Th and Eu which are important for Galactic chronometry (cf. Cowan et al. 1997, 1999). These studies also address the question of the universality of the solar system " $r$-process" abundance pattern (hereafter referred to as the solar $r$-pattern, cf. Cowan et al. 1995, 1996; Sneden et al. 1994, 1996, 1998, 1999; Crawford et al. 1998). In particular, Sneden et al. (1996, 1998, 1999) have demonstrated that the abundances of elements in the Pt peak (at mass number $A \sim 195)$ and down to $\mathrm{Ba}(A \sim 135)$ in $\mathrm{CS} 22892-052([\mathrm{Fe} / \mathrm{H}]=-3.1)$, HD $115444([\mathrm{Fe} / \mathrm{H}]=-2.77)$, and HD $126238([\mathrm{Fe} / \mathrm{H}]=-1.67)$ are in remarkable accord with the solar $r$-pattern.

It has generally been considered that the abundances of the $r$-process nuclei are due to a single generic type of events because the $r$-pattern observed in very metal-poor stars agrees so well with that of the solar system. Two classes of $r$-process calculations have been carried out: both rely on theoretical studies of properties of very neutron-rich nuclei, but one uses simple parametrizations of the astrophysical environment (e.g., Kratz et al. 1993) while the other has a detailed astrophysical context (e.g., Meyer et al. 1992; Takahashi, Witti, \& Janka 1994; Woosley et al. 1994). So far, no stellar model succeeds in generating the conditions required to produce the entire solar $r$-pattern (Witti, Janka, \& Takahashi 1994; Qian \& Woosley 1996; Hoffman, Woosley, \& Qian 1997; Meyer \& Brown 1997; Freiburghaus et al. 1999).

However, it has been shown that the meteoritic data on the inventory of ${ }^{129} \mathrm{I}$ (with a lifetime of $\left.\bar{\tau}_{129}=2.27 \times 10^{7} \mathrm{yr}\right)$ and ${ }^{182} \mathrm{Hf}\left(\bar{\tau}_{182}=1.30 \times 10^{7} \mathrm{yr}\right)$ in the early solar system 
require that the stellar sources responsible for these two nuclei be decoupled (Wasserburg, Busso, \& Gallino 1996, hereafter WBG96). The nuclide ${ }^{129} \mathrm{I}$ is a pure $r$-process product (Cameron 1993). As ${ }^{182} \mathrm{Hf}$ cannot be produced effectively by the $s$-process in Asymptotic Giant Branch stars (Wasserburg et al. 1994; Busso, Gallino, \& Wasserburg 1999), the solar inventory of ${ }^{182} \mathrm{Hf}$ must also have originated from an $r$-process. The meteoritic requirement for diverse $r$-process events can be seen from the following argument.

Let us first assume that there were only a single type of $r$-process events. With the recognition that both ${ }^{127} \mathrm{I}$ and ${ }^{129} \mathrm{I}$ must be produced concurrently and at about the same yields, the observed abundance ratio $\left({ }^{129} \mathrm{I} /{ }^{127} \mathrm{I}\right)_{\mathrm{SSF}}=10^{-4}$ (Reynolds 1960; Jeffrey \& Reynolds 1961; see Brazzle et al. 1999 for a recent summary) at the time of solar system formation (SSF) then demands that the last injection of ${ }^{129} \mathrm{I}$ into the interstellar medium (ISM) from which the solar nebula was formed had taken place $\sim 10^{8} \mathrm{yr}$ earlier (cf. Schramm \& Wasserburg 1970). This result can be obtained by considering two extreme cases for the $r$-process production history prior to SSF: (1) continuous uniform production (CUP, i.e., with an infinitesimal interval between successive events) with the last event occurring $(\delta t)_{\mathrm{CUP}}$ years before SSF, and (2) a single production (SP) event occurring $(\delta t)_{\mathrm{SP}}$ years before SSF. In the CUP case, we have

$$
\left(\frac{{ }^{129} \mathrm{I}}{{ }^{127} \mathrm{I}}\right)_{\mathrm{SSF}}=\left(\frac{Y_{129}}{Y_{127}}\right)\left(\frac{\bar{\tau}_{129}}{T_{\mathrm{UP}}}\right) \exp \left[-(\delta t)_{\mathrm{CUP}} / \bar{\tau}_{129}\right]
$$

where $Y_{A}$ represents the yield of the nuclide " $A$ " in a single event and $T_{\mathrm{UP}}$ is the period of uniform production. For $Y_{129} / Y_{127}=1$ and $T_{\mathrm{UP}}=10^{10} \mathrm{yr}$, equation (1) gives $(\delta t)_{\mathrm{CUP}}=7.09 \times 10^{7} \mathrm{yr}$. In the SP case, we have

$$
\left(\frac{129}{{ }^{127} \mathrm{I}}\right)_{\mathrm{SSF}}=\left(\frac{Y_{129}}{Y_{127}}\right) \exp \left[-(\delta t)_{\mathrm{SP}} / \bar{\tau}_{129}\right]
$$

which gives $(\delta t)_{\mathrm{SP}}=2.09 \times 10^{8} \mathrm{yr}$ for $Y_{129} / Y_{127}=1$. Were there only a single type of $r$-process sources for both ${ }^{129} \mathrm{I}$ and ${ }^{182} \mathrm{Hf}$, the abundance ratio $\left({ }^{182} \mathrm{Hf} /{ }^{180} \mathrm{Hf}\right)_{\mathrm{SSF}}$ (with the 
use of the solar $r$-process abundance of ${ }^{182} \mathrm{~W}$ and the solar abundance of ${ }^{180} \mathrm{Hf}$ given in Table 1) would have decayed to values of $4.27 \times 10^{-8}$ (SP) to $2.28 \times 10^{-6}$ (CUP) on the timescales deduced for the last injection of ${ }^{129} \mathrm{I}$. Such values are in clear conflict with the recently observed value of $\left({ }^{182} \mathrm{Hf} /{ }^{180} \mathrm{Hf}\right)_{\mathrm{SSF}}=2.4 \times 10^{-4}$ in meteorites (Harper \& Jacobsen 1996; Lee \& Halliday 1995, 1996, 1997, 1999). Therefore, the meteoritic data on ${ }^{129} \mathrm{I}$ and ${ }^{182} \mathrm{Hf}$ require at least two distinct types of $r$-process events.

As the solar system abundances represent an average of Galactic chemical evolution on larger scales, it is reasonable to generalize the implications of the meteoritic data discussed above to the larger-scale problem of the "r-process." Using this approach, Wasserburg et al. (WBG96) concluded that there had to be at least two types of $r$-process events, one (H) occurring at a high frequency and one (L) at a low frequency. The recurrence timescale for the high frequency events was shown to be $\sim 10^{7} \mathrm{yr}$, commensurate with the timescale for replenishment of a typical molecular cloud with fresh Type II supernova (hereafter referred to simply as supernova) debris. They further pointed out that there should be distinct differences in the $r$-process abundance peaks in very metal-poor stars relative to the solar $r$-pattern. In particular, they inferred that there would be a dominance of the hypothesized high frequency supernova sources at early times with an abundance excess of the Pt peak at $A \sim 195$ relative to the $A \sim 130$ peak below Ba.

Qian, Vogel \& Wasserburg (1998, hereafter QVW98) have shown that in a twocomponent model to account for the solar $r$-pattern and accommodate the meteoritic data on ${ }^{129} \mathrm{I}$ and ${ }^{182} \mathrm{Hf}$ at the same time, the nuclei between the peak at $A \sim 130$ and the one at $A \sim 195$ are always produced along with the latter peak. In addition, it is not readily possible to produce the $A \sim 130$ peak without substantially populating the region beyond this peak, especially when $\nu_{e}$ capture on nuclei plays a significant role. They also found that the total mass yield of the low frequency supernova L sources responsible for the low 
mass peak at $A \sim 130$ must be $\sim 10$ times that of the high frequency $\mathrm{H}$ sources mainly producing high mass nuclei beyond $A \sim 130$. They further speculated that the difference between the $\mathrm{H}$ and $\mathrm{L}$ sources was associated with the frequent formation of a black hole in supernova $\mathrm{H}$ events (resulting in $\sim 5 \times 10^{8}$ black holes with masses $\sim 1 M_{\odot}$ in the present Galaxy) and the less common production of a neutron star in the $\mathrm{L}$ events (resulting in a present Galactic inventory of $\sim 5 \times 10^{7}$ neutron stars).

As mentioned earlier, stellar abundances of elements associated with both the $A \sim 130$ and $195 r$-process peaks at very low metallicities are under active investigation (e.g., Sneden et al. 1996, 1998, 1999). A preliminary report made by Cowan \& Sneden (1999) suggests that more than one type of $r$-process events may be required. For comparison with stellar observations, it is particularly useful to present specific quantitative inferences for the $r$-process elemental abundances in very metal-poor stars based on the meteoritic data discussed above. It will be shown that a two-component model has specific predictions for both the relative abundance patterns and the absolute abundances of $r$-process elements in stars formed from an ISM seeded with the ejecta from an individual supernova. A criterion for such earliest formed stars will be given based on the Eu abundance. It will further be argued that the $r$-process abundances in many very metal-poor stars are the result of many supernova contributions and that the "metallicity" $[\mathrm{Fe} / \mathrm{H}]$ cannot provide a means of identifying the earliest formed stars.

This paper is organized as follows. In $\S 2$, we show how the characteristics of a two-component $r$-process model, such as the fractions of ${ }^{127} \mathrm{I}$ and ${ }^{182} \mathrm{~W}$ contributed by the $\mathrm{H}$ and $\mathrm{L}$ events to the total solar $r$-process abundances of these two nuclei, are determined by the abundance ratios $\left({ }^{129} \mathrm{I} /{ }^{127} \mathrm{I}\right)_{\mathrm{SSF}}$ and $\left({ }^{182} \mathrm{Hf} /{ }^{180} \mathrm{Hf}\right)_{\mathrm{SSF}}$ in meteorites. Two scenarios for the uniform production of $r$-process nuclei relevant for the solar system abundances are discussed to provide bounds on the proposed model. In $\S 3$, we determine the yields 
in the $\mathrm{H}$ and $\mathrm{L}$ events in our model from the solar $r$-process abundances by choosing the yield templates in the low and high mass regions based on the observed $r$-pattern in very metal-poor stars. We compare these predicted yields with the observed stellar abundances at very low metallicities in $\S 4$ and give our conclusions in $\S 5$. Appendix A contains more general discussion of a two-component $r$-process model, which emphasizes again the well-defined results from this model.

\section{Characteristics of a two-component $r$-process model}

In this paper, we will only consider addition of supernova debris to a "standard" mass of the ISM (see WBG96 and QVW98) without discussing the change in abundances due to astration that will store matter in stars over different timescales. We assume that the solar $r$-pattern is the result of two distinct types of supernovae that occur at different frequencies over a time $T_{\mathrm{UP}}$ in the Galactic history preceding solar system formation (SSF). With the relative supernova yield of ${ }^{235} \mathrm{U}$ to ${ }^{238} \mathrm{U}$ approximated by the ratio of the number of precursors for these two nuclei (cf. Fowler \& Hoyle 1960; Fowler 1961), the estimate of the abundance ratio $\left({ }^{235} \mathrm{U} /{ }^{238} \mathrm{U}\right)_{\mathrm{SSF}}$ based on a uniform rate of nucleosynthesis agrees very well with the observed value of $\left({ }^{235} \mathrm{U} /{ }^{238} \mathrm{U}\right)_{\mathrm{SSF}}=0.317$ (e.g., Qian, Vogel, \& Wasserburg 1999). This applies for uniform production timescales $T_{\mathrm{UP}} \approx 10^{10} \mathrm{yr}$, which are longer than the lifetime of ${ }^{238} \mathrm{U}\left(\bar{\tau}_{238}=6.45 \times 10^{9} \mathrm{yr}\right)$ and much longer than that of ${ }^{235} \mathrm{U}\left(\bar{\tau}_{235}=1.02 \times 10^{9} \mathrm{yr}\right)$. If the actinide production rate is estimated to be of the form $\exp \left(-t / \bar{\tau}_{p}\right)$, we find that the rate changes only by a factor of $\sim 2$ over $10^{10}$ yr (i.e., $\left.\bar{\tau}_{p} \sim 1.3 \times 10^{10} \mathrm{yr}\right)$ in order to account for the observed value of $\left({ }^{235} \mathrm{U} /{ }^{238} \mathrm{U}\right)_{\mathrm{SSF}}$. We thus adopt a model of uniform production (cf. WBG96; Qian et al. 1999).

There are three issues involved in considering the early solar system abundance of short-lived nuclei derived from supernovae. These are the yield in a supernova, the dilution 
factor of the ISM, and the time difference between the last supernova event and the formation of the solar system. We will discuss two scenarios, in which the last supernova event is either somewhat earlier than (scenario A) or coincident with (scenario B, i.e., a trigger for) the formation of the solar system. These two scenarios are considered as bounds to the proposed model.

\subsection{Scenario A}

For simplicity, let us choose a standard dilution factor for all supernovae (cf. Tsujimoto \& Shigeyama 1998; Qian et al. 1999) and focus on a specific volume of the ISM corresponding to the standard mass diluting the debris from an individual supernova. Over a time $T_{\mathrm{UP}}$ in the Galactic history preceding SSF, supernovae in this "volume" can inject fresh nucleosynthesis products to give the abundances of radioactive nuclei in the early solar system and those of stable nuclei in the present solar system (cf. WBG96; QVW98). Let us assume that a supernova $r$-process event regularly occurs every $\Delta$ years in this volume with the last event taking place $\Delta$ years prior to SSF (scenario A). In this scenario, SSF occurs just before a new injection of $r$-process nuclei into the ISM and $\Delta$ years after the most recent injection. Then for a short-lived nuclide " $\mathcal{R}$ " with $\bar{\tau}_{\mathcal{R}} \ll T_{\text {UP }}$, the net number of $\mathcal{R}$ nuclei present at the time of SSF is

$$
N_{\mathcal{R}}\left(t_{\mathrm{SSF}}\right)=\sum_{j=1}^{j_{\max }} Y_{\mathcal{R}} \exp \left(-j \Delta / \bar{\tau}_{\mathcal{R}}\right) \approx Y_{\mathcal{R}} /\left[\exp \left(\Delta / \bar{\tau}_{\mathcal{R}}\right)-1\right]
$$

where $Y_{\mathcal{R}}$ is the number of $\mathcal{R}$ nuclei produced per event (i.e., the yield) and is assumed to be constant. Note that for $\Delta \ll \bar{\tau}_{\mathcal{R}}$, we have

$$
N_{\mathcal{R}}\left(t_{\mathrm{SSF}}\right) \approx Y_{\mathcal{R}} \frac{\bar{\tau}_{\mathcal{R}}}{\Delta}=Y_{\mathcal{R}} \bar{f} \bar{\tau}_{\mathcal{R}}
$$

where $\bar{f}=1 / \Delta$ is the frequency. However, for $\Delta \gg \bar{\tau}_{\mathcal{R}}$, we have $N_{\mathcal{R}}\left(t_{\mathrm{SSF}}\right) \approx Y_{\mathcal{R}} \exp \left(-\Delta / \bar{\tau}_{\mathcal{R}}\right)$ and the last event dominates at SSF. Obviously, for a stable nuclide " $\mathcal{S}$," the number of $\mathcal{S}$ 
nuclei present at the time of SSF is

$$
N_{\mathcal{S}}\left(t_{\mathrm{SSF}}\right)=Y_{\mathcal{S}} \frac{T_{\mathrm{UP}}}{\Delta}
$$

where $Y_{\mathcal{S}}$ is the number of $\mathcal{S}$ nuclei produced per event and should be about the same as the yield of the corresponding short-lived nuclide. We now turn to the case of two distinct $r$-process components.

\subsubsection{The limiting case}

First consider the limiting case where the $\mathrm{L}$ events occurring at a frequency $\bar{f}_{\mathrm{L}}=1 / \Delta_{\mathrm{L}}$ produce all of the ${ }^{129} \mathrm{I}$ and ${ }^{127} \mathrm{I}$ but no ${ }^{182} \mathrm{Hf}$, while the $\mathrm{H}$ events occurring at a frequency $\bar{f}_{\mathrm{H}}=1 / \Delta_{\mathrm{H}}$ produce all of the ${ }^{182} \mathrm{Hf}$ (hence all of the $r$-process contribution to its stable daughter ${ }^{182} \mathrm{~W}$ ) but no ${ }^{129} \mathrm{I}$ or ${ }^{127} \mathrm{I}$. The meaning of $\mathrm{L}$ and $\mathrm{H}$ will become clear shortly. In this limiting case, we have

$$
\left(\frac{{ }^{129} \mathrm{I}}{{ }^{127} \mathrm{I}}\right)_{\mathrm{SSF}}=\left(\frac{Y_{129}}{Y_{127}}\right)\left(\frac{\bar{\tau}_{129}}{T_{\mathrm{UP}}}\right) \frac{\Delta_{\mathrm{L}} / \bar{\tau}_{129}}{\exp \left(\Delta_{\mathrm{L}} / \bar{\tau}_{129}\right)-1},
$$

and

$$
\left(\frac{{ }^{182} \mathrm{Hf}}{{ }^{182} \mathrm{~W}_{r}}\right)_{\mathrm{SSF}}=\left(\frac{\bar{\tau}_{182}}{T_{\mathrm{UP}}}\right) \frac{\Delta_{\mathrm{H}} / \bar{\tau}_{182}}{\exp \left(\Delta_{\mathrm{H}} / \bar{\tau}_{182}\right)-1},
$$

where ${ }^{182} \mathrm{~W}_{r}$ stands for the $r$-process contribution to the solar inventory of ${ }^{182} \mathrm{~W}$. Equations (6) and (7) can be reduced to the form

$$
f\left(X_{129}^{\mathrm{L}}\right)=C_{\mathrm{I}}
$$

and

$$
f\left(X_{182}^{\mathrm{H}}\right)=C_{\mathrm{Hf}},
$$

when we define $X_{129}^{\mathrm{L}} \equiv \Delta_{\mathrm{L}} / \bar{\tau}_{129}, X_{182}^{\mathrm{H}} \equiv \Delta_{\mathrm{H}} / \bar{\tau}_{182}$,

$$
f(X) \equiv \frac{X}{\exp (X)-1}
$$




$$
C_{\mathrm{I}} \equiv \frac{\left({ }^{129} \mathrm{I} /{ }^{127} \mathrm{I}\right)_{\mathrm{SSF}}}{\left(Y_{129} / Y_{127}\right)\left(\bar{\tau}_{129} / T_{\mathrm{UP}}\right)},
$$

and

$$
C_{\mathrm{Hf}} \equiv \frac{\left({ }^{182} \mathrm{Hf} /{ }^{182} \mathrm{~W}_{r}\right)_{\mathrm{SSF}}}{\bar{\tau}_{182} / T_{\mathrm{UP}}}
$$

Both ${ }^{129} \mathrm{I}$ and ${ }^{127} \mathrm{I}$ are essentially pure $r$-process nuclei. As these two nuclei are so close in mass number, the relative yield $Y_{129} / Y_{127}$ in the $\mathrm{L}$ events must be close to unity. For clarity of presentation, we choose $Y_{129} / Y_{127}=1$ in the following discussion. However, we have checked that all of the results obtained in this paper are insensitive to variations of $Y_{129} / Y_{127}$ between 1 and 2 . On the scale of $N_{\odot}(\mathrm{Si})=10^{6}$, the solar abundance of ${ }^{180} \mathrm{Hf}$ is $N_{\odot}\left({ }^{180} \mathrm{Hf}\right)=0.0541$ (Anders \& Grevesse 1989) and the solar $r$-process abundance of ${ }^{182} \mathrm{~W}$ is $N_{\odot, r}\left({ }^{182} \mathrm{~W}\right)=0.0222$ (Käppeler et al. 1991; Arlandini et al. 1999). This gives $\left({ }^{182} \mathrm{Hf} /{ }^{182} \mathrm{~W}_{r}\right)_{\mathrm{SSF}}=5.85 \times 10^{-4}$. With the above information, we obtain $C_{\mathrm{I}}=0.0441$ and $C_{\mathrm{Hf}}=0.450$ for $T_{\mathrm{UP}}=10^{10} \mathrm{yr}$ (assumed throughout this paper). These and other data pertinent to our discussion are summarized in Table 1.

For the numerical values of $C_{\mathrm{I}}$ and $C_{\mathrm{Hf}}$ chosen above, the solutions to equations (8) and (9) give $\Delta_{\mathrm{L}}=1.06 \times 10^{8} \mathrm{yr}$ and $\Delta_{\mathrm{H}}=1.85 \times 10^{7} \mathrm{yr}$. Thus, L can be understood to stand for low frequency and $\mathrm{H}$ for high frequency $r$-process events.

\subsubsection{The general case}

In general, a two-component model does not require that the $\mathrm{H}$ events produce none of the low mass $(A \lesssim 130)$ nuclei associated with ${ }^{127} \mathrm{I}$ or that the $\mathrm{L}$ events produce no high mass $(A>130)$ nuclei associated with ${ }^{182} \mathrm{~W}$. It only requires that the relative yields in these two mass regions be different (cf. QVW98). For the general two-component model, 
equation (6) is replaced by

$$
\left(\frac{{ }^{129} \mathrm{I}}{{ }^{127} \mathrm{I}}\right)_{\mathrm{SSF}}=\left(\frac{Y_{129}^{\mathrm{L}}}{Y_{127}^{\mathrm{L}}}\right)\left(\frac{\bar{\tau}_{129}}{T_{\mathrm{UP}}}\right) \frac{f\left(X_{129}^{\mathrm{H}}\right)+\left(Y_{129}^{\mathrm{L}} / Y_{129}^{\mathrm{H}}\right)\left(\Delta_{\mathrm{H}} / \Delta_{\mathrm{L}}\right) f\left(X_{129}^{\mathrm{L}}\right)}{\left(Y_{127}^{\mathrm{H}} / Y_{127}^{\mathrm{L}}\right)\left(Y_{129}^{\mathrm{L}} / Y_{129}^{\mathrm{H}}\right)+\left(Y_{129}^{\mathrm{L}} / Y_{129}^{\mathrm{H}}\right)\left(\Delta_{\mathrm{H}} / \Delta_{\mathrm{L}}\right)},
$$

where we have defined $X_{129}^{\mathrm{H}} \equiv \Delta_{\mathrm{H}} / \bar{\tau}_{129}$ and $X_{129}^{\mathrm{L}} \equiv \Delta_{\mathrm{L}} / \bar{\tau}_{129}$. If we assume $Y_{129}^{\mathrm{L}} / Y_{127}^{\mathrm{L}}=Y_{129}^{\mathrm{H}} / Y_{127}^{\mathrm{H}}=Y_{129} / Y_{127}$, then equation (13) becomes

$$
\frac{f\left(X_{129}^{\mathrm{H}}\right)+\left(Y_{129}^{\mathrm{L}} / Y_{129}^{\mathrm{H}}\right)\left(\Delta_{\mathrm{H}} / \Delta_{\mathrm{L}}\right) f\left(X_{129}^{\mathrm{L}}\right)}{1+\left(Y_{129}^{\mathrm{L}} / Y_{129}^{\mathrm{H}}\right)\left(\Delta_{\mathrm{H}} / \Delta_{\mathrm{L}}\right)}=C_{\mathrm{I}}
$$

which can be rewritten as

$$
\left(\frac{Y_{129}^{\mathrm{L}}}{Y_{129}^{\mathrm{H}}}\right)\left(\frac{\Delta_{\mathrm{H}}}{\Delta_{\mathrm{L}}}\right)=\left(\frac{Y_{127}^{\mathrm{L}}}{Y_{127}^{\mathrm{H}}}\right)\left(\frac{\Delta_{\mathrm{H}}}{\Delta_{\mathrm{L}}}\right)=\frac{f\left(X_{129}^{\mathrm{H}}\right)-C_{\mathrm{I}}}{C_{\mathrm{I}}-f\left(X_{129}^{\mathrm{L}}\right)} .
$$

Similarly, we obtain

$$
\left(\frac{Y_{182}^{\mathrm{L}}}{Y_{182}^{\mathrm{H}}}\right)\left(\frac{\Delta_{\mathrm{H}}}{\Delta_{\mathrm{L}}}\right)=\frac{f\left(X_{182}^{\mathrm{H}}\right)-C_{\mathrm{Hf}}}{C_{\mathrm{Hf}}-f\left(X_{182}^{\mathrm{L}}\right)}
$$

where we have defined $X_{182}^{\mathrm{H}} \equiv \Delta_{\mathrm{H}} / \bar{\tau}_{182}$ and $X_{182}^{\mathrm{L}} \equiv \Delta_{\mathrm{L}} / \bar{\tau}_{182}$.

By definition, we have $\Delta_{\mathrm{H}}<\Delta_{\mathrm{L}}$. Physical solutions (i.e., positive values for the yield ratios $Y_{127}^{\mathrm{L}} / Y_{127}^{\mathrm{H}}$ and $Y_{182}^{\mathrm{L}} / Y_{182}^{\mathrm{H}}$ ) to equations (15) and (16) then require $\Delta_{\mathrm{L}}>1.06 \times 10^{8} \mathrm{yr} \equiv \Delta_{\mathrm{L}}^{\min }$ and $\Delta_{\mathrm{H}}<1.85 \times 10^{7} \mathrm{yr} \equiv \Delta_{\mathrm{H}}^{\max }\left(\right.$ hence $\left.\Delta_{\mathrm{H}} / \Delta_{\mathrm{L}}<0.175\right)$. Note that $\Delta_{\mathrm{L}}^{\min }$ and $\Delta_{\mathrm{H}}^{\max }$ correspond to the values of $\Delta_{\mathrm{L}}$ and $\Delta_{\mathrm{H}}$ in the limiting case. The interrelationship between $\Delta_{\mathrm{H}}$ and $\Delta_{\mathrm{L}}$ and the yield ratios $Y_{127}^{\mathrm{L}} / Y_{127}^{\mathrm{H}}$ and $Y_{182}^{\mathrm{L}} / Y_{182}^{\mathrm{H}}$ can be understood as follows. The production of ${ }^{129} \mathrm{I}$ and ${ }^{127} \mathrm{I}$ in the $\mathrm{H}$ events mainly increases the inventory of the radioactive ${ }^{129} \mathrm{I}$ at $\mathrm{SSF}$ as the last few $\mathrm{H}$ events occurred much closer to the formation of the early solar system than the last L event. In order to satisfy the observed value of $\left({ }^{129} \mathrm{I} /{ }^{127} \mathrm{I}\right)_{\mathrm{SSF}}$, the last $\mathrm{L}$ event must then move to even earlier times, i.e., $\Delta_{\mathrm{L}}$ must increase from that in the limiting case. On the other hand, the production of any ${ }^{182} \mathrm{Hf}$ (hence ${ }^{182} \mathrm{~W}$ ) in the L events mainly increase the stable inventory of ${ }^{182} \mathrm{~W}_{r}$ at SSF. In order to satisfy the abundance ratio $\left({ }^{182} \mathrm{Hf} /{ }^{182} \mathrm{~W}_{r}\right)_{\mathrm{SSF}}$, this then requires a decrease in $\Delta_{\mathrm{H}}$ from that in the limiting case to allow more contribution to the radioactive ${ }^{182} \mathrm{Hf}$ from the last 
few $\mathrm{H}$ events to compensate for the increased portion of the ${ }^{182} \mathrm{~W}_{r}$ inventory present from the L events.

In addition to equations (15) and (16), a further requirement of the two-component model is that the integrated $r$-process production of the stable $\mathcal{S}$ nuclei must satisfy

$$
\frac{N_{127}^{\mathrm{H}}\left(t_{\mathrm{SSF}}\right)+N_{127}^{\mathrm{L}}\left(t_{\mathrm{SSF}}\right)}{N_{182}^{\mathrm{H}}\left(t_{\mathrm{SSF}}\right)+N_{182}^{\mathrm{L}}\left(t_{\mathrm{SSF}}\right)}=\left(\frac{Y_{127}^{\mathrm{H}}}{Y_{182}^{\mathrm{H}}}\right) \frac{1+\left(Y_{127}^{\mathrm{L}} / Y_{127}^{\mathrm{H}}\right)\left(\Delta_{\mathrm{H}} / \Delta_{\mathrm{L}}\right)}{1+\left(Y_{182}^{\mathrm{L}} / Y_{182}^{\mathrm{H}}\right)\left(\Delta_{\mathrm{H}} / \Delta_{\mathrm{L}}\right)}=\frac{N_{\odot, r}\left({ }^{127} \mathrm{I}\right)}{N_{\odot, r}\left({ }^{182} \mathrm{~W}\right)},
$$

where $N_{\odot, r}\left({ }^{127} \mathrm{I}\right) \approx N_{\odot}\left({ }^{127} \mathrm{I}\right)$ and $N_{\odot, r}\left({ }^{182} \mathrm{~W}\right)$ are the solar $r$-process abundances of ${ }^{127} \mathrm{I}$ and ${ }^{182} \mathrm{~W}$ [given in Table 1 on the scale of $N_{\odot}(\mathrm{Si})=10^{6}$ ], respectively.

The characteristics of scenario A are fully specified by equations (15)-(17). For convenience in discussing the solutions to these equations, we define

$$
F_{r}^{\mathrm{L}}\left({ }^{127} \mathrm{I}\right) \equiv \frac{N_{127}^{\mathrm{L}}\left(t_{\mathrm{SSF}}\right)}{N_{127}^{\mathrm{H}}\left(t_{\mathrm{SSF}}\right)+N_{127}^{\mathrm{L}}\left(t_{\mathrm{SSF}}\right)}=1-\frac{1}{1+\left(Y_{127}^{\mathrm{L}} / Y_{127}^{\mathrm{H}}\right)\left(\Delta_{\mathrm{H}} / \Delta_{\mathrm{L}}\right)},
$$

which is the fraction of ${ }^{127} \mathrm{I}$ contributed by the $\mathrm{L}$ events to the corresponding total solar $r$-process abundance, and

$$
F_{r}^{\mathrm{H}}\left({ }^{182} \mathrm{~W}\right) \equiv \frac{N_{182}^{\mathrm{H}}\left(t_{\mathrm{SSF}}\right)}{N_{182}^{\mathrm{H}}\left(t_{\mathrm{SSF}}\right)+N_{182}^{\mathrm{L}}\left(t_{\mathrm{SSF}}\right)}=\frac{1}{1+\left(Y_{182}^{\mathrm{L}} / Y_{182}^{\mathrm{H}}\right)\left(\Delta_{\mathrm{H}} / \Delta_{\mathrm{L}}\right)},
$$

which is the fraction of ${ }^{182} \mathrm{~W}$ contributed by the $\mathrm{H}$ events to the corresponding total solar $r$-process abundance. The limiting cases outlined in $\S 2.1 .1$ correspond to $F_{r}^{\mathrm{L}}\left({ }^{127} \mathrm{I}\right)=1$, for which no ${ }^{129} \mathrm{I}$ or ${ }^{127} \mathrm{I}$ is produced in the $\mathrm{H}$ events, and $F_{r}^{\mathrm{H}}\left({ }^{182} \mathrm{~W}\right)=1$, for which no ${ }^{182} \mathrm{Hf}$ (hence no ${ }^{182} \mathrm{~W}$ ) is produced in the $\mathrm{L}$ events. We also define

$$
U_{\mathrm{H}} \equiv \frac{Y_{127}^{\mathrm{H}} / Y_{182}^{\mathrm{H}}}{N_{\odot, r}\left({ }^{127} \mathrm{I}\right) / N_{\odot, r}\left({ }^{182} \mathrm{~W}\right)},
$$

which measures the relative yield of ${ }^{127} \mathrm{I}$ to ${ }^{182} \mathrm{~W}$ in an $\mathrm{H}$ event with respect to the corresponding solar $r$-process abundance ratio. When $U_{\mathrm{H}}<1$, the production of ${ }^{127} \mathrm{I}$ relative to ${ }^{182} \mathrm{~W}$ in an $\mathrm{H}$ event is depleted (subsolar) compared with the solar $r$-pattern. Note that similarly defined quantities can be obtained as

$$
\left.F_{r}^{\mathrm{H}}\left({ }^{127} \mathrm{I}\right)\right) \equiv \frac{N_{127}^{\mathrm{H}}\left(t_{\mathrm{SSF}}\right)}{N_{127}^{\mathrm{H}}\left(t_{\mathrm{SSF}}\right)+N_{127}^{\mathrm{L}}\left(t_{\mathrm{SSF}}\right)}=1-F_{r}^{\mathrm{L}}\left({ }^{127} \mathrm{I}\right),
$$




$$
F_{r}^{\mathrm{L}}\left({ }^{182} \mathrm{~W}\right) \equiv \frac{N_{182}^{\mathrm{L}}\left(t_{\mathrm{SSF}}\right)}{N_{182}^{\mathrm{H}}\left(t_{\mathrm{SSF}}\right)+N_{182}^{\mathrm{L}}\left(t_{\mathrm{SSF}}\right)}=1-F_{r}^{\mathrm{H}}\left({ }^{182} \mathrm{~W}\right),
$$

and

$$
U_{\mathrm{L}} \equiv \frac{Y_{127}^{\mathrm{L}} / Y_{182}^{\mathrm{L}}}{N_{\odot, r}\left({ }^{127} \mathrm{I}\right) / N_{\odot, r}(182 \mathrm{~W})}=U_{\mathrm{H}} \frac{\left(Y_{127}^{\mathrm{L}} / Y_{127}^{\mathrm{H}}\right)}{\left(Y_{182}^{\mathrm{L}} / Y_{182}^{\mathrm{H}}\right)}
$$

A unique solution for $\Delta_{\mathrm{H}}, \Delta_{\mathrm{L}}, Y_{127}^{\mathrm{L}} / Y_{127}^{\mathrm{H}}, Y_{182}^{\mathrm{L}} / Y_{182}^{\mathrm{H}}$, and $Y_{127}^{\mathrm{H}} / Y_{182}^{\mathrm{H}}$ is not possible to obtain from equations (15)-(17). However, substantial restrictions on all of these parameters that exhibit the basic characteristics of scenario A of the two-component model can be obtained by only considering the constraints $\Delta_{\mathrm{H}}<\Delta_{\mathrm{H}}^{\max }=1.85 \times 10^{7}$ yr and $\Delta_{\mathrm{L}}>\Delta_{\mathrm{L}}^{\mathrm{min}}=1.06 \times 10^{8}$ yr on equations (15) and (16) (see Appendix A). We will now use a clear example for our discussion of this scenario. We assume that $\Delta_{\mathrm{H}} / \Delta_{\mathrm{L}}=0.1$, which specifies a physical range of $(1.06$ to 1.85$) \times 10^{8}$ yr for $\Delta_{\mathrm{L}}$. The key parameters $U_{\mathrm{H}}, U_{\mathrm{L}}$, $F_{r}^{\mathrm{L}}\left({ }^{127} \mathrm{I}\right)$, and $F_{r}^{\mathrm{H}}\left({ }^{182} \mathrm{~W}\right)$ obtained for this example are shown in Figure 1 as functions of $\Delta_{\mathrm{L}}$. The relative yield of ${ }^{127} \mathrm{I}$ to ${ }^{182} \mathrm{~W}$ in the $\mathrm{L}$ events is 3.28 times the corresponding solar $r$-process abundance ratio $\left(U_{\mathrm{L}}=3.28\right.$, i.e., supersolar $)$ at $\Delta_{\mathrm{L}}=\Delta_{\mathrm{L}}^{\min }=1.06 \times 10^{8}$ yr and increases as the limiting case $\Delta_{\mathrm{H}}=\Delta_{\mathrm{H}}^{\max }$ is approached. By contrast, the relative yield of ${ }^{127} \mathrm{I}$ to ${ }^{182} \mathrm{~W}$ in the $\mathrm{H}$ events is always more than a factor of 15 less than the corresponding solar $r$-process abundance ratio $\left(U_{\mathrm{H}} \leq 0.065\right.$, i.e., subsolar $)$. For $\Delta_{\mathrm{L}}=1.85 \times 10^{8}$ yr corresponding to $\Delta_{\mathrm{H}}=\Delta_{\mathrm{H}}^{\max }, 93.5 \%$ of the ${ }^{127} \mathrm{I}$ is produced by the $\mathrm{L}$ events $\left[F_{r}^{\mathrm{L}}\left({ }^{127} \mathrm{I}\right)=0.935\right]$ and all of the solar $r$-process ${ }^{182} \mathrm{~W}$ is produced by the $\mathrm{H}$ events $\left[F_{r}^{\mathrm{H}}\left({ }^{182} \mathrm{~W}\right)=1\right]$. For $\Delta_{\mathrm{L}}=\Delta_{\mathrm{L}}^{\min }$, all of the ${ }^{127} \mathrm{I}$ is produced by the $\mathrm{L}$ events $\left[F_{r}^{\mathrm{L}}\left({ }^{127} \mathrm{I}\right)=1\right]$ and $69.5 \%$ of the solar $r$-process ${ }^{182} \mathrm{~W}$ is produced by the $\mathrm{H}$ events $\left[F_{r}^{\mathrm{H}}\left({ }^{182} \mathrm{~W}\right)=0.695\right]$. Note that in the above example for scenario $\mathrm{A}$, the fraction of ${ }^{127} \mathrm{I}$ contributed by the $\mathrm{H}$ events to the corresponding total solar $r$-process abundance is at most $6.5 \%\left[F_{r}^{\mathrm{H}}\left({ }^{127} \mathrm{I}\right) \leq 0.065\right]$. On the other hand, the fraction of ${ }^{182} \mathrm{~W}$ contributed by the $\mathrm{L}$ events to the corresponding total solar $r$-process abundance is $F_{r}^{\mathrm{L}}\left({ }^{182} \mathrm{~W}\right) \geq 0.1$ for $\Delta_{\mathrm{L}}<1.63 \times 10^{8} \mathrm{yr}$ and can be as much as $30.5 \%$ at $\Delta_{\mathrm{L}}=\Delta_{\mathrm{L}}^{\min }$. This means that for most of the physical $\Delta_{\mathrm{L}}$ values corresponding to $\Delta_{\mathrm{H}} / \Delta_{\mathrm{L}}=0.1$ in scenario 
A, the yields of ${ }^{182} \mathrm{~W}$ in the $\mathrm{H}$ and $\mathrm{L}$ events are comparable, i.e., $Y_{182}^{\mathrm{L}} / Y_{182}^{\mathrm{H}} \sim 1$.

In deriving the above results on ${ }^{127} \mathrm{I}$, we have assumed that $Y_{129}^{\mathrm{H}} / Y_{127}^{\mathrm{H}}=Y_{129}^{\mathrm{L}} / Y_{127}^{\mathrm{L}}(\mathrm{cf}$. eqs. [13] and [14]). We note that $Y_{129}^{\mathrm{H}} / Y_{127}^{\mathrm{H}}$ may differ from $Y_{129}^{\mathrm{L}} / Y_{127}^{\mathrm{L}}$. For example, the yields for $A \lesssim 130$ in the $\mathrm{H}$ events may drop steeply at smaller $A$ (cf. QVW98). Consider the extreme case where $Y_{127}^{\mathrm{H}}=0$, for which the denominator on the right-hand side of equation (13) would be $\left(Y_{129}^{\mathrm{L}} / Y_{129}^{\mathrm{H}}\right)\left(\Delta_{\mathrm{H}} / \Delta_{\mathrm{L}}\right)$. The correct equation to be used in this case would be identical to equation (14) except for the unity term in the denominator. However, it is clear that practically all of the low mass nuclei are produced in the L events, i.e., $\left(Y_{129}^{\mathrm{L}} / Y_{129}^{\mathrm{H}}\right)\left(\Delta_{\mathrm{H}} / \Delta_{\mathrm{L}}\right) \gg 1$. Therefore, our assumption of $Y_{129}^{\mathrm{H}} / Y_{127}^{\mathrm{H}}=Y_{129}^{\mathrm{L}} / Y_{127}^{\mathrm{L}}$ has little effect on estimating the production of low mass nuclei in the L events. On the other hand, the ratio $Y_{127}^{\mathrm{L}} / Y_{127}^{\mathrm{H}}$ derived from equation (15) may be regarded as a safe lower limit for $Y_{A}^{\mathrm{L}} / Y_{A}^{\mathrm{H}}$ at $A<130$, although we will later use it to represent the actual yield ratios in the low mass region.

\subsection{Scenario B}

We now discuss the second scenario which differs from scenario A in that the solar system is assumed to have been formed immediately after the last $\mathrm{H}$ event (scenario B). The standard dilution factor of the ISM is assumed for the debris from this last event. At

the time of SSF in scenario B, the net number of short-lived $\mathcal{R}$ nuclei due to the $\mathrm{H}$ events is

$$
N_{\mathcal{R}}^{\mathrm{H}}\left(t_{\mathrm{SSF}}\right)=\sum_{j=0}^{j_{\max }} Y_{\mathcal{R}}^{\mathrm{H}} \exp \left(-j \Delta / \bar{\tau}_{\mathcal{R}}\right) \approx Y_{\mathcal{R}}^{\mathrm{H}} /\left[1-\exp \left(-\Delta / \bar{\tau}_{\mathcal{R}}\right)\right] .
$$

Accordingly, we define

$$
g(X) \equiv \frac{X}{1-\exp (-X)},
$$

and replace equations (15) and (16) by

$$
\left(\frac{Y_{127}^{\mathrm{L}}}{Y_{127}^{\mathrm{H}}}\right)\left(\frac{\Delta_{\mathrm{H}}}{\Delta_{\mathrm{L}}}\right)=\frac{g\left(X_{129}^{\mathrm{H}}\right)-C_{\mathrm{I}}}{C_{\mathrm{I}}-f\left(X_{129}^{\mathrm{L}}\right)},
$$


and

$$
\left(\frac{Y_{182}^{\mathrm{L}}}{Y_{182}^{\mathrm{H}}}\right)\left(\frac{\Delta_{\mathrm{H}}}{\Delta_{\mathrm{L}}}\right)=\frac{g\left(X_{182}^{\mathrm{H}}\right)-C_{\mathrm{Hf}}}{C_{\mathrm{Hf}}-f\left(X_{182}^{\mathrm{L}}\right)},
$$

respectively. The characteristics of scenario B are then fully specified by equations (26), (27), and (17).

As $g(X) \geq 1$ for $X \geq 0$, physical solutions to equations (26) and (27) only require that $\Delta_{\mathrm{L}}>\Delta_{\mathrm{L}}^{\min }=1.06 \times 10^{8} \mathrm{yr}$. Unlike in scenario A, there is no restriction on $\Delta_{\mathrm{H}}$ in scenario B. However, some general characteristics of this scenario can still be obtained without any detailed knowledge of $\Delta_{\mathrm{H}}$. As $f\left(X_{129}^{\mathrm{L}}\right)>0$, equation (26) gives

$$
1+\left(\frac{Y_{127}^{\mathrm{L}}}{Y_{127}^{\mathrm{H}}}\right)\left(\frac{\Delta_{\mathrm{H}}}{\Delta_{\mathrm{L}}}\right)>\frac{g\left(X_{129}^{\mathrm{H}}\right)}{C_{\mathrm{I}}} \geq \frac{1}{C_{\mathrm{I}}},
$$

which means that $F_{r}^{\mathrm{H}}\left({ }^{127} \mathrm{I}\right)<C_{\mathrm{I}}=0.044$ and $F_{r}^{\mathrm{L}}\left({ }^{127} \mathrm{I}\right)>0.956$. Thus the $\mathrm{H}$ events provide less than $4.4 \%$ of the solar $r$-process ${ }^{127} \mathrm{I}$ in scenario B.

For $\Delta_{\mathrm{L}}>\Delta_{\mathrm{L}}^{\min }=1.06 \times 10^{8} \mathrm{yr}$, we have $f\left(X_{182}^{\mathrm{L}}\right)<2.35 \times 10^{-3} \ll C_{\mathrm{Hf}}$. Equation (27) then gives

$$
1+\left(\frac{Y_{182}^{\mathrm{L}}}{Y_{182}^{\mathrm{H}}}\right)\left(\frac{\Delta_{\mathrm{H}}}{\Delta_{\mathrm{L}}}\right) \approx \frac{g\left(X_{182}^{\mathrm{H}}\right)}{C_{\mathrm{Hf}}} \geq \frac{1}{C_{\mathrm{Hf}}},
$$

which means that $F_{r}^{\mathrm{H}}\left({ }^{182} \mathrm{~W}\right) \lesssim C_{\mathrm{Hf}}=0.45$ and $F_{r}^{\mathrm{L}}\left({ }^{182} \mathrm{~W}\right) \gtrsim 0.55$. Therefore, the L events account for essentially all of the solar $r$-process ${ }^{127} \mathrm{I}$ and more than $55 \%$ of the solar $r$-process ${ }^{182} \mathrm{~W}$ in scenario B.

To give a specific example for scenario $B$, we again choose $\Delta_{H} / \Delta_{L}=0.1$ and further impose $\Delta_{\mathrm{L}} \leq 2 \times 10^{8}$ yr [near the $(\delta t)_{\mathrm{SP}}$ value for the single production case in eq. (2)]. The key parameters $U_{\mathrm{H}}, U_{\mathrm{L}}, F_{r}^{\mathrm{L}}\left({ }^{127} \mathrm{I}\right)$, and $F_{r}^{\mathrm{L}}\left({ }^{182} \mathrm{~W}\right)$ obtained for this example are shown as functions of $\Delta_{\mathrm{L}}$ in Figure 2. In accord with the above general discussion of scenario B, the $\mathrm{L}$ events in this example provide (100 to 97$) \%$ of the solar $r$-process ${ }^{127} \mathrm{I}\left[F_{r}^{\mathrm{L}}\left({ }^{127} \mathrm{I}\right)=1\right.$ to $0.97]$ and (69 to 77$) \%$ of the solar $r$-process ${ }^{182} \mathrm{~W}\left[F_{r}^{\mathrm{L}}\left({ }^{182} \mathrm{~W}\right)=0.69\right.$ to 0.77$]$ over the range of $(1.06$ to 2$) \times 10^{8} \mathrm{yr}$ for $\Delta_{\mathrm{L}}$. As a result, the relative yield of ${ }^{127} \mathrm{I}$ to ${ }^{182} \mathrm{~W}$ in an $\mathrm{L}$ event in 
scenario B is very close to the corresponding solar $r$-process abundance ratio $\left(U_{\mathrm{L}}=1.44\right.$ to 1.26 in Fig. 2).

\section{Yields in a two-component $r$-process model}

Let us now consider the yields in individual $\mathrm{H}$ and $\mathrm{L}$ events in a two-component $r$-process model. For a stable nuclide $\mathcal{S}$, the total $r$-process abundance of $\mathcal{S}$ nuclei at the time of SSF is

$$
N_{\mathcal{S}}\left(t_{\mathrm{SSF}}\right)=Y_{\mathcal{S}}^{\mathrm{H}} \frac{T_{\mathrm{UP}}}{\Delta_{\mathrm{H}}}+Y_{\mathcal{S}}^{\mathrm{L}} \frac{T_{\mathrm{UP}}}{\Delta_{\mathrm{L}}}=\frac{Y_{\mathcal{S}}^{\mathrm{H}}}{F_{r}^{\mathrm{H}}(\mathcal{S})}\left(\frac{T_{\mathrm{UP}}}{\Delta_{\mathrm{H}}}\right)
$$

where as in $\S 2$, the parameter

$$
F_{r}^{\mathrm{H}}(\mathcal{S})=\frac{1}{1+\left(Y_{\mathcal{S}}^{\mathrm{L}} / Y_{\mathcal{S}}^{\mathrm{H}}\right)\left(\Delta_{\mathrm{H}} / \Delta_{\mathrm{L}}\right)}
$$

is the fraction of the nuclide $\mathcal{S}$ contributed by the $\mathrm{H}$ events to the total solar $r$-process abundance of $\mathcal{S}$ nuclei. Assuming that the diluting number of hydrogen $(\mathcal{H})$ atoms per event is a constant $N_{\mathcal{H}}$, we have $N_{\mathcal{S}}\left(t_{\mathrm{SSF}}\right) / N_{\mathcal{H}}=N_{\odot, r}(\mathcal{S}) / N_{\odot}(\mathcal{H})$ and

$$
\log \left(\frac{Y_{\mathcal{S}}^{\mathrm{H}}}{N_{\mathcal{H}}}\right)=\log \left[\frac{N_{\odot, r}(\mathcal{S})}{N_{\odot}(\mathcal{H})}\right]+\log \left[F_{r}^{\mathrm{H}}(\mathcal{S})\right]-\log \left(\frac{T_{\mathrm{UP}}}{\Delta_{\mathrm{H}}}\right)
$$

where $N_{\odot, r}(\mathcal{S})$ is the solar $r$-process abundance of $\mathcal{S}$ nuclei and $N_{\odot}(\mathcal{H})$ is the solar abundance of hydrogen. For a star formed from an ISM contaminated by a single H event, its abundance of $\mathcal{S}$ nuclei (with respect to hydrogen) is given by equation (32). In standard spectroscopic notation, $\log \epsilon(\mathcal{S})=\log (\mathcal{S} / \mathcal{H})+12$, where $\mathcal{S} / \mathcal{H}$ is the abundance ratio of the

nuclide $\mathcal{S}$ to hydrogen in the star. So equation (32) for the $\mathrm{H}$ event can be rewritten as

$$
\log \epsilon_{\mathrm{H}}(\mathcal{S})=\log \epsilon_{\odot, r}(\mathcal{S})+\log \left[F_{r}^{\mathrm{H}}(\mathcal{S})\right]-\log \left(T_{\mathrm{UP}} / \Delta_{\mathrm{H}}\right)
$$

Similarly, for the L event, we obtain

$$
\log \epsilon_{\mathrm{L}}(\mathcal{S})=\log \epsilon_{\odot, r}(\mathcal{S})+\log \left[F_{r}^{\mathrm{L}}(\mathcal{S})\right]-\log \left(T_{\mathrm{UP}} / \Delta_{\mathrm{L}}\right)
$$


where $F_{r}^{\mathrm{L}}(\mathcal{S})=1-F_{r}^{\mathrm{H}}(\mathcal{S})$ is the fraction of the nuclide $\mathcal{S}$ contributed by the L events to the total solar $r$-process abundance of $\mathcal{S}$ nuclei. Note that when put in terms of the $\log \epsilon$ values for a star formed from an ISM contaminated by a single $\mathrm{H}$ or L event, the yields of $\mathcal{S}$ nuclei as given in equations (33) and (34) only depend on the basic results $F_{r}^{\mathrm{H}}(\mathcal{S})$ [or equivalently, $\left.F_{r}^{\mathrm{L}}(\mathcal{S})\right], T_{\mathrm{UP}} / \Delta_{\mathrm{H}}$, and $T_{\mathrm{UP}} / \Delta_{\mathrm{L}}$ from a two-component $r$-process model.

\subsection{Yields in a simplified two-component model}

With equations (33) and (34), we can now relate the yields in a two-component $r$-process model, and hence the abundances to be observed in stars formed from an ISM contaminated by a single $\mathrm{H}$ or $\mathrm{L}$ event, to the solar $r$-process abundances. The application to ${ }^{127} \mathrm{I}$ and ${ }^{182} \mathrm{~W}$ is straightforward as their yields are a direct consequence of the model involving the radioactive nuclei ${ }^{129} \mathrm{I}$ and ${ }^{182} \mathrm{Hf}$ discussed in $\S 2$. In Figure 3, we show $\delta \log \epsilon_{\mathrm{H}}(\mathcal{S}) \equiv \log \epsilon_{\mathrm{H}}(\mathcal{S})-\log \epsilon_{\odot, r}(\mathcal{S})$ and $\delta \log \epsilon_{\mathrm{L}}(\mathcal{S}) \equiv \log \epsilon_{\mathrm{L}}(\mathcal{S})-\log \epsilon_{\odot, r}(\mathcal{S})$ for ${ }^{127} \mathrm{I}$ and ${ }^{182} \mathrm{~W}$ as functions of $\Delta_{\mathrm{L}}$. The corresponding parameters $F_{r}^{\mathrm{H}}(\mathcal{S})$ and $F_{r}^{\mathrm{L}}(\mathcal{S})$ shown in Figures $1(\mathrm{~b})$ and $2(\mathrm{~b})$ have been used to obtain the curves in Figure 3. Note that $\delta \log \epsilon_{\mathrm{H}}\left({ }^{182} \mathrm{~W}\right)$ is dominated by the term $-\log \left(T_{\mathrm{UP}} / \Delta_{\mathrm{H}}\right)$ in equation (33), and $\delta \log \epsilon_{\mathrm{L}}\left({ }^{127} \mathrm{I}\right)$ is dominated by the term $-\log \left(T_{\mathrm{UP}} / \Delta_{\mathrm{L}}\right)$ in equation (34). For $\Delta_{\mathrm{H}} / \Delta_{\mathrm{L}}=0.1$ (assumed in Figures 1 and 2) and $\Delta_{\mathrm{L}}=1.5 \times 10^{8} \mathrm{yr}$, the contributions from these two terms are -2.82 and -1.82 , respectively. These are the minimum values of $\delta \log \epsilon_{\mathrm{H}}\left({ }^{182} \mathrm{~W}\right)$ and $\delta \log \epsilon_{\mathrm{L}}\left({ }^{127} \mathrm{I}\right)$ to be

expected for the assumed values of $\Delta_{\mathrm{H}}$ and $\Delta_{\mathrm{L}}$ as $F_{r}^{\mathrm{H}}\left({ }^{182} \mathrm{~W}\right)$ and $F_{r}^{\mathrm{L}}\left({ }^{127} \mathrm{I}\right)$ cannot exceed unity (cf. eqs. 33 and 34).

If one wishes to establish the yields of stable nuclei other than ${ }^{127} \mathrm{I}$ and ${ }^{182} \mathrm{~W}$, then it is necessary to know the ratios $Y_{\mathcal{S}}^{\mathrm{L}} / Y_{\mathcal{S}}^{\mathrm{H}}$ of their yields in an L event to those in an $\mathrm{H}$ event (cf. eq. 30]). The simplest approach is to assume that the H and L events produce the low mass nuclei associated with ${ }^{127} \mathrm{I}$ and the high mass nuclei associated with ${ }^{182} \mathrm{~W}$ in 
different proportions, but with each mass region having exactly the same yield template as the corresponding solar $r$-pattern. In this case, it is only necessary to define the two mass regions. The yield ratios $Y_{\mathcal{S}}^{\mathrm{L}} / Y_{\mathcal{S}}^{\mathrm{H}}$ for all the stable nuclei in the low or high mass region are then specified by $Y_{127}^{\mathrm{L}} / Y_{127}^{\mathrm{H}}$ or $Y_{182}^{\mathrm{L}} / Y_{182}^{\mathrm{H}}$, respectively. Likewise, the values of $\delta \log \epsilon_{\mathrm{H}}(\mathcal{S})$ and $\delta \log \epsilon_{\mathrm{L}}(\mathcal{S})$ for all the stable nuclei in the low or high mass region (cf. eqs. 31], 33, and [34]) are specified by the corresponding values for ${ }^{127} \mathrm{I}$ or ${ }^{182} \mathrm{~W}$ (shown in Fig. 3), respectively.

We choose to assign nuclei with $A \leq 130$, including ${ }^{127} \mathrm{I}$, to the low mass region and those with $A>130$, including ${ }^{182} \mathrm{~W}$, to the high mass region. This choice is justified by both the observed stellar abundances at very low metallicities and the nuclear physics of the r-process. Observational studies by Sneden et al. (1996, 1998, 1999) have established that the $r$-process elemental abundance pattern for $\mathrm{Ba}(A \sim 135)$ and higher atomic numbers in very metal-poor stars agrees very well with the corresponding solar $r$-pattern. In two cases, Th was also detected and after correction for the age of the star, the observed abundance of Th relative to those of the stable $r$-process nuclei at lower atomic numbers (e.g., Eu) is again consistent with the solar $r$-pattern (cf. Cowan et al. 1999). These studies include stars with metallicities of $[\mathrm{Fe} / \mathrm{H}]=-1.67$ down to -3.1 although the data sets are not complete over the entire range of atomic numbers corresponding to $A>130$. In addition, the extensive work by McWilliam et al. (1995) and McWilliam (1998) has shown that the abundance ratio of $\mathrm{Ba}$ to $\mathrm{Eu}(A \sim 151)$ at very low metallicities is remarkably well-behaved and lies in a very narrow band around the corresponding solar $r$-process value (see $\S 4$ and Fig. 9). Therefore, it is reasonable to conclude from these observational studies that apart from some small discrepancies (e.g., at Ce, see Sneden et al. 1998 and Fig. 8), the yield pattern for $A>130$ in all $r$-process events is very close to the solar $r$-pattern in this mass region. 
Further, the above observational result can be understood from the nuclear physics of the $r$-process. Consider the making of the Pt peak at $A \sim 195$ by rapid neutron capture onto seed nuclei with $A \lesssim 100$. During the $r$-process, the nuclear flow is mainly controlled by $\beta$-decay of the progenitor nuclei. The time needed for the nuclear flow to clear a mass region is approximately the sum of $\beta$-decay lifetimes for the progenitor nuclei in this mass region. Before reaching the progenitor nuclei with the $N=126$ closed neutron shell that will produce the Pt peak after exhaustion of all the neutrons, the nuclear flow must pass through the progenitor nuclei at $A \sim 130$ with the $N=82$ closed neutron shell. If the sum of $\beta$-decay lifetimes for the $N=82$ progenitor nuclei is at least comparable to that for the progenitor nuclei with $82<N \leq 126$, then the building-up of the Pt peak is always controlled by the gradual diminishing of the $N=82$ nuclei at $A \sim 130$, and a quasi-steady $\beta$-decay equilibrium will result to largely determine the yield pattern in the $130<A \lesssim 195$ mass region (see, e.g., Kratz et al. 1993). This quasi-steady $\beta$-decay equilibrium may well explain the observational result that the $r$-process elemental abundance pattern for $\mathrm{Ba}$ and higher atomic numbers in very metal-poor stars is remarkably close to the corresponding solar $r$-pattern. Accordingly, we define $A>130$ as the high mass region and $A \leq 130$ as the low mass region. The lower end of the $A \leq 130$ region is not quite clear as it depends on, e.g., the mass number of the seed nuclei for the $r$-process, but we will assume it to be around ${ }^{100}$ Mo.

The solar $r$-process nuclear abundances (Käppeler et al. 1989, 1991) are shown in terms of $\log \epsilon_{\odot, r}(A)$ in Figure 4(a). The vertical dashed line in this figure indicates the boundary $(A=130)$ between the low and high mass regions chosen in our simplified two-component model. As discussed above, we will use $Y_{127}^{\mathrm{L}} / Y_{127}^{\mathrm{H}}$ or $Y_{182}^{\mathrm{L}} / Y_{182}^{\mathrm{H}}$ to fix the yield ratios $Y_{\mathcal{S}}^{\mathrm{L}} / Y_{\mathcal{S}}^{\mathrm{H}}$ for all the stable nuclei in the $A \leq 130$ or $A>130$ mass region, respectively. Consequently, we can obtain the yields of all the stable nuclei in the $\mathrm{H}$ and $\mathrm{L}$ events from the solar $r$-process nuclear abundances shown in Figure $4(\mathrm{a})$ using the $\delta \log \epsilon_{\mathrm{H}}(\mathcal{S})$ and 
$\delta \log \epsilon_{\mathrm{L}}(\mathcal{S})$ values for ${ }^{127} \mathrm{I}$ and ${ }^{182} \mathrm{~W}$ shown in Figure 3. However, for comparison with stellar observations, we have to use the elemental abundances. The solar $r$-process elemental abundances in the low and high mass regions are shown in terms of $\log \epsilon_{\odot, r}(Z)$ in Figure 4(b). As Xe (at atomic number $Z=54$ ) has $r$-process isotopes in both the low and high mass regions, the vertical dashed line indicating the boundary between the two mass regions is put at $Z=54$ in Figure $4(\mathrm{~b})$. The elements $\mathrm{Ba}$ and $\mathrm{Eu}$ in the high $(A>130)$ mass region are commonly observed for very metal-poor stars (e.g., McWilliam et al. 1995) and observations of $\mathrm{Pd}, \mathrm{Ag}$, and $\mathrm{Cd}$ in the low $(A \leq 130)$ mass region are being actively pursued for such stars (Sneden et al. 1999). The solar $r$-process abundances $N_{\odot, r}$ and the corresponding $\log \epsilon_{\odot, r}$ values of these elements are given in Table 2. For comparison with observational data, we will focus on $\mathrm{Ag}(A \sim 107)$ and $\mathrm{Eu}$ as the representative elements of the low and high mass regions, respectively.

In Figure 5, we show $\log \epsilon_{\mathrm{H}}(\mathrm{Ag}), \log \epsilon_{\mathrm{L}}(\mathrm{Ag}), \log \epsilon_{\mathrm{H}}(\mathrm{Eu})$, and $\log \epsilon_{\mathrm{L}}(\mathrm{Eu})$ as functions of $\Delta_{\mathrm{L}}$. As discussed above, these elemental yields are obtained from the solar $r$-process elemental abundances shown in Figure $4(\mathrm{~b})$ with the use of the $\delta \log \epsilon_{\mathrm{H}}(\mathcal{S})$ and $\delta \log \epsilon_{\mathrm{L}}(\mathcal{S})$ values for ${ }^{127} \mathrm{I}$ and ${ }^{182} \mathrm{~W}$ shown in Figure 3. In scenario A [with the assumption of $\Delta_{\mathrm{H}} / \Delta_{\mathrm{L}}=0.1$, cf. Fig. 5(a)], typical yields are $\log \epsilon_{\mathrm{L}}(\mathrm{Ag}) \approx-0.65$ and $\log \epsilon_{\mathrm{H}}(\mathrm{Eu}) \approx-2.38$ (with variations $\sim \pm 0.1$ and $\sim \pm 0.2$, respectively) over the narrow physical range for $\Delta_{\mathrm{L}}$. In addition, we have $\log \epsilon_{\mathrm{H}}(\mathrm{Ag}) \approx-3.3$ to -2.7 and $\log \epsilon_{\mathrm{L}}(\mathrm{Eu}) \approx-2.4$ to -2.0 in regions of $\Delta_{\mathrm{L}}$ away from the limiting cases of $\Delta_{\mathrm{L}}^{\min }$ or $\Delta_{\mathrm{H}}^{\max }$, respectively. In scenario $\mathrm{B}$ [with the assumption of $\Delta_{\mathrm{H}} / \Delta_{\mathrm{L}}=0.1$, cf. Fig. 5(b)], typical yields are $\log \epsilon_{\mathrm{L}}(\mathrm{Ag}) \approx-0.64$, $\log \epsilon_{\mathrm{H}}(\mathrm{Eu}) \approx-2.89$, and $\log \epsilon_{\mathrm{L}}(\mathrm{Eu}) \approx-1.45$, with variations $\sim \pm(0.1$ to 0.2$)$ over the range of $(1.06$ to 2$) \times 10^{8} \mathrm{yr}$ for $\Delta_{\mathrm{L}}$. Away from the limiting case of $\Delta_{\mathrm{L}}=\Delta_{\mathrm{L}}^{\min }, \log \epsilon_{\mathrm{H}}(\mathrm{Ag})$ also has a rather constant value $\sim-3.3$. The results for the specific case of $\Delta_{\mathrm{L}}=1.5 \times 10^{8} \mathrm{yr}$ and $\Delta_{\mathrm{H}} / \Delta_{\mathrm{L}}=0.1$ are typical of scenarios $\mathrm{A}$ and $\mathrm{B}$, and are given in Table 3 . 


\subsection{Yield patterns and total mass yields in the simplified two-component model}

Using the case of $\Delta_{\mathrm{L}}=1.5 \times 10^{8}$ yr and $\Delta_{\mathrm{H}} / \Delta_{\mathrm{L}}=0.1$ for both scenarios A and B as examples, we show the yield patterns in the above simplified two-component model in Figures 6 and 7. For comparison with stellar observations, the elemental yields shown in these two figures are given in terms of the $\log \epsilon$ values for a star formed from an ISM contaminated by a single $\mathrm{H}$ or $\mathrm{L}$ event. As in Figure 4, the vertical dashed line at $Z=54$ (Xe) in Figures 6 and 7 indicates the boundary $(A=130)$ between the low and high mass regions in the simplified model. Note that the yield patterns in the $\mathrm{H}$ events are quite similar in the chosen examples for scenarios A and B [cf. Figs. 6(a) and 7(a)]. The downward shifts in $\log \epsilon$ from scenario A to B are 0.3 and 0.5 dex in the low and high mass regions, respectively. However, while the yields in the low mass region in the L events are essentially the same in these examples, the yields in the high mass region increase by 0.7 dex in $\log \epsilon$ from scenario A to B [cf. Figs. 6(b) and 7(b)].

The dotted curve labelled "TS" in Figures 6 and 7 shows the solar $r$-pattern translated to match the yield of $\mathrm{Eu}$ (hence those of $Z>54$ by the assumption of the simplified model) in the chosen examples. With respect to the translated solar $r$-pattern in these examples for scenarios $\mathrm{A}$ and $\mathrm{B}$, the $\mathrm{H}$ event shows a depletion by 1.2 and 1.0 dex, respectively, while the L event shows an enhancement by 0.8 and $0.1 \mathrm{dex}$, respectively, of the yields below Xe. Note that in the example for scenario B, the gross yield pattern in the L event almost coincides with the translated solar $r$-pattern.

To characterize the depletion of the yields in the low mass region (below Xe) relative to those in the high mass region in the $\mathrm{H}$ event with respect to the solar $r$-pattern, we define

$$
[\mathrm{Ag} / \mathrm{Eu}]_{r}^{\mathrm{H}} \equiv \log \left(\frac{Y_{\mathrm{Ag}}^{\mathrm{H}}}{Y_{\mathrm{Eu}}^{\mathrm{H}}}\right)-\log \left[\frac{N_{\odot, r}(\mathrm{Ag})}{N_{\odot, r}(\mathrm{Eu})}\right]=\delta \log \epsilon_{\mathrm{H}}(\mathrm{Ag})-\delta \log \epsilon_{\mathrm{H}}(\mathrm{Eu}) .
$$


In the simplified model, we have $\delta \log \epsilon_{\mathrm{H}}(\mathrm{Ag})=\delta \log \epsilon_{\mathrm{H}}\left({ }^{127} \mathrm{I}\right)$ and $\delta \log \epsilon_{\mathrm{H}}(\mathrm{Eu})=$ $\delta \log \epsilon_{\mathrm{H}}\left({ }^{182} \mathrm{~W}\right)$. The values of $[\mathrm{Ag} / \mathrm{Eu}]_{r}^{\mathrm{H}}$ and the similarly defined $[\mathrm{Ag} / \mathrm{Eu}]_{r}^{\mathrm{L}}$ are given in Table 3 for the case of $\Delta_{\mathrm{L}}=1.5 \times 10^{8}$ yr and $\Delta_{\mathrm{H}} / \Delta_{\mathrm{L}}=0.1$ for both scenarios A and B.

We are also interested in comparing the total mass yields in the $\mathrm{H}$ and $\mathrm{L}$ events. The ratio of the total mass yield in an $\mathrm{L}$ event to that in an $\mathrm{H}$ event is

$$
m \approx \frac{\sum_{A=100}^{209} Y_{A}^{\mathrm{L}}}{\sum_{A=100}^{209} Y_{A}^{\mathrm{H}}},
$$

where we have neglected the small yields of the actinides $(A>209)$ in both the $\mathrm{H}$ and $\mathrm{L}$ events. For the specific examples shown in Figures 6 and 7 , we have $m \approx 12$ for scenario A [cf. Figs. 6(a) and (b)] and $m \approx 58$ for scenario B [cf. Figs. 7(a) and (b)]. These values are typical of the two scenarios. In $\S 5$, we will briefly describe a possible mechanism that can cause the total $r$-process yields from different supernova sources to vary by factors $\gtrsim 10$ (cf. QVW98). However, we note here that larger differences in the total yields (e.g., $m \approx 58$ ) may be more difficult to produce.

\section{Comparison with observed abundances in very metal-poor stars}

We now turn to the comparison of the yields in a single $\mathrm{H}$ or $\mathrm{L}$ event in the above simplified two-component $r$-process model with the observed abundances in very metal-poor stars using $\log \epsilon(\mathrm{Eu})$ as a guide. In the examples where $\Delta_{\mathrm{H}} / \Delta_{\mathrm{L}}=0.1$ is assumed, we have $\log \epsilon_{\mathrm{H}}(\mathrm{Eu})=-2.62$ to -2.22 over the entire physical range for $\Delta_{\mathrm{L}}$ and $\log \epsilon_{\mathrm{L}}(\mathrm{Eu})=-2.4$ to -2.0 over most of this range in scenario $\mathrm{A}$, while in scenario $\mathrm{B}$, we have $\log \epsilon_{\mathrm{H}}(\mathrm{Eu})=-2.98$ to -2.83 and $\log \epsilon_{\mathrm{L}}(\mathrm{Eu})=-1.62$ to -1.30 over the range of (1.06 to 2$) \times 10^{8} \mathrm{yr}$ for $\Delta_{\mathrm{L}}$ (cf. Fig. 5). The observed values of $\log \epsilon(\mathrm{Eu})$ for four very metal-poor stars (Sneden et al. 1996, 1998, 1999) are given in Table 4. By comparing the $\log \epsilon(\mathrm{Eu})$ values from our model with the observed ones, we can infer whether a single $\mathrm{H}$ or L event in scenario A or B can explain 
the data reasonably well. As indicated in Table 4, our inferences from such a comparison are: (1) for HD 122563, a single $\mathrm{H}$ or $\mathrm{L}$ event in scenario $\mathrm{A}$ is compatible with the observed $\log \epsilon(\mathrm{Eu})$ value, while in scenario $\mathrm{B}$, a single $\mathrm{H}$ event is only marginally compatible and a single L event is incompatible with the observation; (2) for HD 115444, only a single L event in scenario B is compatible with the observation; and (3) for CS 22892-052 with the lowest metallicity and HD 126238 with the highest metallicity of the four stars shown in Table 4, we find that no single event is compatible with the data.

The comparison of the yields in a single event in our model with the observed abundances in HD 122563 is shown in Figure 8(a). The dotted curve labelled "TS" in this figure is the solar $r$-pattern translated to match the observed Eu abundance. In contrast, the solid curve labelled "H (A)" or the dashed curve labelled "L (A)" shows the yields in an $\mathrm{H}$ or L event in scenario A [cf. Figs. 6(a) or (b)] obtained directly from our model (i.e., no fitting is attempted). As we can see from Figure 8(a), the agreement between the solid curve and the data is very good while the dashed curve describes the data less well. However, a decisive test for the agreement between our model and the data is the Ag abundance. It is predicted that the Ag abundance to be measured in HD 122563 should be either higher by 0.8 dex $[\mathrm{L}(\mathrm{A})]$ or lower by over 1 dex $[\mathrm{H}(\mathrm{A})]$ than the value corresponding to the translated solar $r$-pattern. In Figure $8(\mathrm{~b})$, the yields in an L event in scenario B obtained from our model [cf. Fig. 7(b)] are shown by the solid curve labelled "L (B)," and can be compared with the observed abundances in HD 115444. The solar $r$-pattern translated to match the observed Eu abundance (not shown) is almost identical to the solid curve. We note the good agreement between the solid curve and the data, and emphasize that a decisive test of this agreement is again the Ag abundance to be measured in HD 115444.

It can be seen that our model does not describe the observations very well (cf. Table 4) 
if the abundances in very metal-poor stars indeed represent the result of a single supernova precursor contaminating the ISM with a constant dilution factor. It is also clear from the observations alone that in order to account for the data, a single supernova precursor model for the abundances in very metal-poor stars has to invoke large variations in supernova production of $\mathrm{Fe}$ and $\mathrm{Eu}$, or otherwise requires a grossly heterogeneous distribution of $\mathrm{Fe}$ relative to the heavy $r$-process nuclei in the mixing of the supernova debris with the ISM. As shown in Table 4, HD 122563 and HD 115444 have essentially the same $\log \epsilon(\mathrm{Fe})$ value but differ in $\log \epsilon(\mathrm{Eu})$ by about 1 dex, while CS22892-052 and HD 126238 have essentially the same $\log \epsilon(\mathrm{Eu})$ value but differ in $\log \epsilon(\mathrm{Fe})$ by about 1.4 dex. In addition, although CS22892-052 has the lowest $\log \epsilon(\mathrm{Fe})$ value of the four stars shown in Table 4, its $\log \epsilon(\mathrm{Eu})$ value is higher than that of HD 122563 by about 1.5 dex. Using additional and more extensive data from McWilliam et al. (1995), we show the $\log \epsilon(\mathrm{Eu})$ and $\log \epsilon(\mathrm{Fe})$ values for 15 very metal-poor stars in Figure 9. Of these stars, two have $[\mathrm{Fe} / \mathrm{H}]=-2.06$ and -1.67 , respectively, and the rest have $[\mathrm{Fe} / \mathrm{H}]=-3.1$ to -2.41 . In contrast to the well-behaved $\mathrm{Ba} / \mathrm{Eu}$ abundance ratio (close to the corresponding solar $r$-process value, cf. McWilliam et al. 1995; McWilliam 1998) shown in the upper part of Figure 9, there is a large dispersion in $\log \epsilon(\mathrm{Eu})$ over the range of $\log \epsilon(\mathrm{Fe})$ for the stellar sample with a corresponding large dispersion in the $\mathrm{Eu} / \mathrm{Fe}$ abundance ratio.

Using similar data and the framework of a single supernova precursor model, Tsujimoto \& Shigeyama (1998) have attributed the large dispersion in the $\mathrm{Eu} / \mathrm{Fe}$ abundance ratio at very low metallicities to the different dependences of Eu and Fe yields on the main sequence mass of the supernova (see also Ishimaru \& Wanajo 1999). In addition, a stochastic chemical evolution model for the early Galaxy has been proposed to explain a similar dispersion in the Ba/Fe abundance ratio (McWilliam 1997, 1998; McWilliam \& Searle 1999). In this alternative model, individual regions are chemically enriched by random sampling of all possible supernova yields through the occurrence of local supernovae. Studies of the 
problem of $\mathrm{Ba}$ enrichment in the Galaxy by means of numerical simulations have been carried out by Raiteri et al. (1999) (see also Travaglio et al. 1999). These numerical studies attribute the large dispersion in the $\mathrm{Ba} / \mathrm{Fe}$ abundance ratio at very low metallicities to the inhomogeneous chemical composition of the ISM from which the stars were formed. While there may be numerous explanations for the large dispersion in log $\epsilon(\mathrm{Eu})$ over the range of $\log \epsilon(\mathrm{Fe})$ for very metal-poor stars, one possibility is that even at metallicities of $[\mathrm{Fe} / \mathrm{H}]$ $\sim-3.0$, the ISM may have already been contaminated by more than a single supernova source. We will consider this possibility using our two-component $r$-process model in the following discussion.

We first discuss the abundances of $r$-process elements in a star formed from an ISM contaminated by a mixture of $n_{\mathrm{H}} \mathrm{H}$ events and $n_{\mathrm{L}} \mathrm{L}$ events. In this case, the Ag and Eu abundances in the star are given by

$$
10^{\log \epsilon(\mathrm{Ag})}=n_{\mathrm{H}} \times 10^{\log \epsilon_{\mathrm{H}}(\mathrm{Ag})}+n_{\mathrm{L}} \times 10^{\log \epsilon_{\mathrm{L}}(\mathrm{Ag})},
$$

and

$$
10^{\log \epsilon(\mathrm{Eu})}=n_{\mathrm{H}} \times 10^{\log \epsilon_{\mathrm{H}}(\mathrm{Eu})}+n_{\mathrm{L}} \times 10^{\log \epsilon_{\mathrm{L}}(\mathrm{Eu})},
$$

respectively. Let us focus on the case of $\Delta_{\mathrm{L}}=1.5 \times 10^{8} \mathrm{yr}$ and $\Delta_{\mathrm{H}} / \Delta_{\mathrm{L}}=0.1$ for scenario A (cf. Table 3). In order to account for the observed Eu abundance of $\log \epsilon(\mathrm{Eu})=-1.53$ in $\mathrm{HD} 115444$ by pure $\mathrm{H}$ events, we need $n_{\mathrm{H}}=7$, which predicts an $\mathrm{Ag}$ abundance of $\log \epsilon(\mathrm{Ag})=-2.08$ for this star. For the assumed values of $\Delta_{\mathrm{H}}$ and $\Delta_{\mathrm{L}}$, the mixture of $n_{\mathrm{H}}=7$ and $n_{\mathrm{L}}=0$ would occur in a standard diluting mass of the ISM with a Poisson probability $P\left(n_{\mathrm{H}}=7, n_{\mathrm{L}}=0\right)=7.4 \%$ over the first $1.05 \times 10^{8}$ yr after the birth of the Galaxy. We note that a second mixture of $n_{\mathrm{H}}=7$ and $n_{\mathrm{L}}=1$ would occur equally likely in the same amount of ISM over the same period of time with a Poisson probability $P\left(n_{\mathrm{H}}=7, n_{\mathrm{L}}=1\right)=5.2 \%$. While the second mixture gives $\log \epsilon(\mathrm{Eu})=-1.44$ in accord with the observed value in HD 115444 , it predicts a much higher $\mathrm{Ag}$ abundance of $\log \epsilon(\mathrm{Ag})=-0.63$ for this star. The 
abundance pattern for the mixture of seven pure H events is shown in Figure 10(a) together with the data on HD 115444. The abundance pattern for the mixture of seven $\mathrm{H}$ events and one L event (not shown) is almost identical to the solar $r$-pattern translated to match the observed Eu abundance (dotted curve labelled "TS" in this figure).

For CS 22892-052 and HD 126238, we first note that the observed Eu abundances are high compared with the yields in any single event (see Tables 3 and 4). We can account for the Eu data on these two stars with a mixture of 27 pure $\mathrm{H}$ events again using the case of $\Delta_{\mathrm{L}}=1.5 \times 10^{8} \mathrm{yr}$ and $\Delta_{\mathrm{H}} / \Delta_{\mathrm{L}}=0.1$ for scenario A. However, the value of $\log \epsilon(\mathrm{Ag})=-1.50$ given by this mixture is far below the preliminary value of $\log \epsilon(\mathrm{Ag}) \approx-0.75 \pm 0.25$ observed in CS 22892-052 (Cowan \& Sneden 1999) while the comparison for HD 126238 cannot be made yet. On the other hand, the mixture of $n_{\mathrm{H}}=26$ and $n_{\mathrm{L}}=1$, which gives $\log \epsilon(\mathrm{Ag})=-0.59$ and $\log \epsilon(\mathrm{Eu})=-0.94$, fits the observations of CS 22892-052, and would occur in a standard diluting mass of the ISM with a Poisson probability $P\left(n_{\mathrm{H}}=26, n_{\mathrm{L}}=1\right)=1.5 \%$ over the first $3.9 \times 10^{8}$ yr after the birth of the Galaxy. The abundance patterns for both the mixture of $n_{\mathrm{H}}=27$ and $n_{\mathrm{L}}=0$ and that of $n_{\mathrm{H}}=26$ and $n_{\mathrm{L}}=1$ are shown in Figure 10(b) together with the data on CS 22892-052. Note that the preliminary Ag data clearly indicate a non-solar $r$-pattern in this star. In addition to revealing possible deviations of the $r$-pattern in very metal-poor stars from that in the solar system, future observations of the abundances of $\mathrm{Ag}$ and other elements in the low mass region in HD 115444 and HD 126238 will further test whether mixtures of multiple supernova $r$-process events in the context of our two-component model may be a viable explanation.

We next discuss the Fe abundance. An estimate of the Fe yield in a single $\mathrm{H}$ or $\mathrm{L}$ event can be obtained under the assumption that $Y_{\mathrm{Fe}}^{\mathrm{H}} \approx Y_{\mathrm{Fe}}^{\mathrm{L}}$ and $\Delta_{\mathrm{H}} / \Delta_{\mathrm{L}}=0.1$. Following the 
derivation of the $r$-process yields (eqs. [33] and [34), we obtain

$$
\log \epsilon_{\mathrm{H}}(\mathrm{Fe}) \approx \log \epsilon_{\mathrm{L}}(\mathrm{Fe}) \approx \log \epsilon_{\odot}(\mathrm{Fe})+\log \alpha-\log \left(\frac{T_{\mathrm{UP}}}{\Delta_{\mathrm{H}}}\right)
$$

where $\alpha$ is the fraction of Fe contributed by Type II supernovae (the sources for the $r$-process nuclei in our model) to the solar abundance of $\mathrm{Fe}\left[\log \epsilon_{\odot}(\mathrm{Fe})=7.51\right.$, Anders \& Grevesse 1989]. For $\alpha \approx 1 / 3$ (cf. Timmes, Woosley, \& Weaver 1995) and $\Delta_{\mathrm{H}}=1.5 \times 10^{7} \mathrm{yr}$ $\left(\Delta_{\mathrm{L}}=1.5 \times 10^{8} \mathrm{yr}\right)$, we have $\log \epsilon_{\mathrm{H}}(\mathrm{Fe}) \approx \log \epsilon_{\mathrm{L}}(\mathrm{Fe}) \approx 4.2$. Inspection of Table 4 shows that except for CS 22892-052, all the three other stars would require a mixture of supernova events to account for their $\log \epsilon(\mathrm{Fe})$ values. This contradicts the requirements from the $\log \epsilon(\mathrm{Eu})$ values in HD 122563 (single event) and CS 22892-052 (mixture). For the mixtures given above to account for the $\log \epsilon(\mathrm{Eu})$ values in $\mathrm{HD} 115444\left(n_{\mathrm{H}}+n_{\mathrm{L}}=7\right.$ or 8$)$ and HD $126238\left(n_{\mathrm{H}}+n_{\mathrm{L}}=27\right)$, we have $\log \epsilon(\mathrm{Fe}) \approx 5.1(\mathrm{HD} 115444)$ and 5.6 (HD 126238). Agreement with the data is obtained only for HD 126238. The poor representation of the data by a model assuming a constant supernova Fe yield is not surprising. Theoretical estimates (Timmes et al. 1995) show that the Fe yields are very sensitive to the main sequence mass of the supernova at all metallicities, and may range from no to high production at zero metallicity. Therefore, the "metallicity" as determined by $[\mathrm{Fe} / \mathrm{H}]$ is at best only a rough guide to time in the early history of the Galaxy. It is not unreasonable for a star such as CS 22892-052 with a very low [Fe/H] value to have been formed from an ISM contaminated by $\sim 10$ supernova $r$-process events. We emphasize that if the timescale for replenishment of typical molecular clouds with fresh $r$-process debris is $\sim 10^{7} \mathrm{yr}$, then the time resolution required to identify the earliest formed stars enriched in $r$-process elements is extremely fine $\left(\Delta_{\mathrm{H}} / T_{\mathrm{UP}} \sim 10^{-3}\right)$. Therefore, the use of a rough chronometer such as $[\mathrm{Fe} / \mathrm{H}]$ cannot define the relevant time periods.

From the remarkable regularity in the $r$-pattern for $\mathrm{Ba}$ and higher atomic numbers observed in very metal-poor stars (Sneden et al. 1996, 1998, 1999), it is reasonable 
to assume that the Eu abundance is a direct measure of the abundances in the high $(A>130)$ mass region. Correspondingly, the Ag abundance may be taken as a direct measure of the abundances in the low $(A \leq 130)$ mass region. With these assumptions, our two-component $r$-process model gives specific predictions for $\log \epsilon_{\mathrm{H}}(\mathrm{Eu})$ and $\log \epsilon_{\mathrm{L}}(\mathrm{Ag})$, and reasonably specific predictions for $\log \epsilon_{\mathrm{H}}(\mathrm{Ag})$ and $\log \epsilon_{\mathrm{L}}(\mathrm{Eu})$ for both scenarios $\mathrm{A}$ and $\mathrm{B}$. This suggests that the proper measure of the age of very metal-poor stars can be defined by $\log \epsilon(\mathrm{Eu})$ rather than $[\mathrm{Fe} / \mathrm{H}]$. We infer that stars formed from an ISM contaminated by a single $\mathrm{H}$ event would have $\log \epsilon_{\mathrm{H}}(\mathrm{Eu})=-2.98$ to -2.22 while those formed from an ISM contaminated by a single $\mathrm{L}$ event would have the less restricted range of $\log \epsilon_{\mathrm{L}}(\mathrm{Eu}) \approx-2.4$ to -1.3 . To make a specific assignment of an $\mathrm{H}$ or $\mathrm{L}$ event to a star will depend on concurrent measurements of abundances in both the low and high mass regions. As the $\mathrm{H}$ events are much more frequent, stars formed from an ISM contaminated by a pure H event would be easier to find, while those formed from an ISM contaminated by the less frequent $\mathrm{L}$ events should contain the debris from the $\mathrm{H}$ events that most likely preceded them.

Based on the typical values of $\log \epsilon_{\mathrm{H}}(\mathrm{Eu})$ and $\log \epsilon_{\mathrm{L}}(\mathrm{Eu})$ given in Table 3 and inspection of Table 4 and Figure 9, we infer that many of the very metal-poor stars studied so far would have to be assigned to formation from an ISM contaminated by multiple $r$-process events. For a constant supernova Fe yield, the values of $\log \epsilon(\mathrm{Fe})$ for these stars also cannot be explained by a single supernova precursor. In any case, the observed values of $\log \epsilon(\mathrm{Fe})$ are not correlated with those of $\log \epsilon(\mathrm{Eu})$ at very low metallicities. However, as noted previously, theoretical estimates of supernova Fe yields are widely variable and using $[\mathrm{Fe} / \mathrm{H}]$ as a measure of the age of very metal-poor stars is highly problematic. To account for the observations with our two-component $r$-process model, we are obliged to consider that Fe yields are not strongly coupled with the production of the $r$-process elements in Type II supernovae at very low metallicities. It is also conceivable that there is an unidentified additional source of Fe at very early times. 


\section{Conclusions}

We have shown the general consequences of a phenomenological two-component $r$-process model based on the ${ }^{129} \mathrm{I}$ and ${ }^{182} \mathrm{Hf}$ abundances in the early solar system. This model assumes a standard mass of the ISM for dilution of the debris from an individual supernova. Two scenarios have been investigated to provide bounds on the model. The frequencies of the $\mathrm{H}\left(\bar{f}_{\mathrm{H}}=1 / \Delta_{\mathrm{H}}\right)$ and $\mathrm{L}\left(\bar{f}_{\mathrm{L}}=1 / \Delta_{\mathrm{L}}\right)$ events proposed in the model are constrained by the meteoritic data on ${ }^{129} \mathrm{I}$ and ${ }^{182} \mathrm{Hf}$. The yields in a single $\mathrm{H}$ or L event are determined from these meteoritic data and the solar $r$-process abundances under the assumption that the yield template in the low $(A \leq 130)$ or high $(A>130)$ mass region is the same for both the $\mathrm{H}$ and $\mathrm{L}$ events and follows the corresponding solar $r$-pattern in each mass region. These yields are represented by the $\log \epsilon$ values for a star formed from an ISM contaminated by a single $\mathrm{H}$ or L event (cf. Figs. 6 and 7). In this approach, the Eu abundance in a single $\mathrm{H}$ event is well defined (cf. Fig. 5). With the addition of subsequent supernova $r$-process debris to the ISM, the abundances from further mixtures of multiple $\mathrm{H}$ and $\mathrm{L}$ events can be obtained in a straightforward manner. This leads to rather explicit quantitative predictions for stellar abundances in the early Galaxy and for the contrast between the stellar abundance pattern at early times and the solar $r$-pattern. These predictions may be directly tested by comparison with the observed abundances in the low and high mass regions in very metal-poor stars.

It is well known that $[\mathrm{Fe} / \mathrm{H}]$ is not a reliable estimator of the Galactic age. Considering the observed Eu abundances, we find that even at very low metallicities of $[\mathrm{Fe} / \mathrm{H}] \sim-3.0$, the ISM may have already been contaminated by many $r$-process events. Therefore, we propose that the abundance of Eu be the criterion for identifying the earliest stars formed in the Galaxy. We predict that those stars with $\log \epsilon(\mathrm{Eu})=-2.98$ to -2.22 were formed

from an ISM contaminated most likely by a single $\mathrm{H}$ event within the first $\sim 10^{7} \mathrm{yr}$ of the 
Galactic history and should have an $\mathrm{Ag} / \mathrm{Eu}$ abundance ratio less than the corresponding solar $r$-process value by a factor of at least 10 . The crucial test for these predictions will again be the measurement of abundances in very metal-poor stars. We recognize that measurements at the low Eu abundances indicated here may pose very difficult observational problems.

In this paper, the fundamental problems of the evolution of supernovae and the sites of the $r$-process have not been addressed. Our approach has been purely phenomenological. If we assume that the production of all the $r$-process nuclei is associated with a proto-typical general supernova event, then a scenario may be suggested that might unify the $H$ and L events (cf. QVW98). We consider that a supernova in its earlier stages ejects matter from the proto-neutron star for the $r$-process with a relatively high number of neutrons per seed nucleus (i.e., a relatively high neutron-to-seed ratio). The $r$-process then dominantly produces nuclei in the high mass region with relatively few residual nuclei in the low mass region. These events are usually (i.e., at a high frequency) terminated by collapse of the proto-neutron star into a black hole, as may be described by the scenario of Brown \& Bethe (1994). On occasion (i.e., at a low frequency), collapse into a black hole does not occur and the ejection of matter for the $r$-process continues but with a lower neutron-to-seed ratio. The $r$-process then dominantly produces nuclei in the low mass region with significant yields also for nuclei in the high mass region. The total mass yield of $r$-process nuclei in the rare events is much higher due to the longer duration of mass ejection from the stable neutron star. The relative production of black holes to neutron stars in supernovae is $\sim 10: 1$ to account for the ratio of frequencies for the $\mathrm{H}$ and $\mathrm{L}$ events. However, the physics that might be responsible for the scenario sketched above remains to be explored.

This work is dedicated to David Norman Schramm and is in the spirit of excitement, hypotheses, and observation that typified his approach. One of us remembers participating 
in the early wonders of nuclear cosmochronology and the search for extinct nuclei during his thesis. The other remembers the dense presentations and mysteries of earlier nuclear cosmochronologic reports and the interest and excitement of the new studies. The approach used here seeks to follow that of previous scholars. "The true method of experience first lights the candle (by hypothesis), and then by means of the candle shows the way, commencing as it does with experience duly ordered ... and from it educing axioms ('first fruits,' provisional conclusions), and from established axioms again new experiments ... Experiment itself shall judge." — Francis Bacon, Novum Organum (1620)

We greatly appreciate the support by John Cowan and Christopher Sneden in freely providing us information on their work and in maintaining a continued level of interest in testing alternative models, however speculative. Discussions with Andrew McWilliam on abundances in very metal-poor stars were of considerable aid. We thank Petr Vogel for comments on an earlier draft of the paper. This work was supported in part by the US Department of Energy under contract W-7405-ENG-36 and grant DE-FG03-88ER-13851, and by NASA under grant NAG 5-4076, Caltech Division Contribution No. 8641(1032). Y.-Z. Q. was supported by the J. Robert Oppenheimer Fellowship at Los Alamos National Laboratory. 


\section{A. General discussion of scenarios A and B}

Without the assumption of $\Delta_{\mathrm{H}} / \Delta_{\mathrm{L}}=0.1$, some general results for scenario $\mathrm{A}$ can still be obtained by considering the restrictions $\Delta_{\mathrm{H}}<\Delta_{\mathrm{H}}^{\max }=1.85 \times 10^{7} \mathrm{yr}$ and $\Delta_{\mathrm{L}}>\Delta_{\mathrm{L}}^{\min }=1.06 \times 10^{8}$ yr on equations (15) and (16). As $f\left(X_{129}^{\mathrm{L}}\right)>0$ and $f\left(X_{129}^{\mathrm{H}}\right)>f\left(\Delta_{\mathrm{H}}^{\max } / \bar{\tau}_{129}\right)=0.646$, equation (15) gives

$$
1+\left(\frac{Y_{127}^{\mathrm{L}}}{Y_{127}^{\mathrm{H}}}\right)\left(\frac{\Delta_{\mathrm{H}}}{\Delta_{\mathrm{L}}}\right)>\frac{f\left(X_{129}^{\mathrm{H}}\right)}{C_{\mathrm{I}}}>\frac{0.646}{C_{\mathrm{I}}},
$$

which means that the fractions of ${ }^{127}$ I contributed by the $\mathrm{H}$ and $\mathrm{L}$ events to the corresponding total solar $r$-process abundance (cf. eqs. [18] and [21]) are $F_{r}^{\mathrm{H}}\left({ }^{127} \mathrm{I}\right)<C_{\mathrm{I}} / 0.646=0.068$ and $F_{r}^{\mathrm{L}}\left({ }^{127} \mathrm{I}\right)>0.932$, respectively.

For $\Delta_{\mathrm{L}}>\Delta_{\mathrm{L}}^{\min }=1.06 \times 10^{8} \mathrm{yr}$, we have $f\left(X_{182}^{\mathrm{L}}\right)<2.35 \times 10^{-3} \ll C_{\mathrm{Hf}}$. Equation (16) then gives

$$
1+\left(\frac{Y_{182}^{\mathrm{L}}}{Y_{182}^{\mathrm{H}}}\right)\left(\frac{\Delta_{\mathrm{H}}}{\Delta_{\mathrm{L}}}\right) \approx \frac{f\left(X_{182}^{\mathrm{H}}\right)}{C_{\mathrm{Hf}}} \leq \frac{1}{C_{\mathrm{Hf}}}
$$

which means that the fractions of ${ }^{182} \mathrm{~W}$ contributed by the $\mathrm{H}$ and $\mathrm{L}$ events to the corresponding total solar $r$-process abundance (cf. eqs. [19] and [22]) are $F_{r}^{\mathrm{H}}\left({ }^{182} \mathrm{~W}\right) \gtrsim C_{\mathrm{Hf}}=0.45$ and $F_{r}^{\mathrm{L}}\left({ }^{182} \mathrm{~W}\right) \lesssim 0.55$, respectively. Therefore, the $\mathrm{L}$ events account

for essentially all of the solar $r$-process ${ }^{127} \mathrm{I}$ and the $\mathrm{H}$ events account for more than $45 \%$ of the solar $r$-process ${ }^{182} \mathrm{~W}$ in scenario $\mathrm{A}$.

Following the general discussion of scenario A presented above and that of scenario B presented in $§ 2.2$, we can obtain some general results on the yields of $\mathrm{Ag}$ and $\mathrm{Eu}$ in the simplified two-component $r$-process model without assuming that $\Delta_{\mathrm{H}} / \Delta_{\mathrm{L}}=0.1$. These results are best represented by $\log \epsilon_{\mathrm{L}}(\mathrm{Ag}), \log \epsilon_{\mathrm{H}}(\mathrm{Eu}),[\mathrm{Ag} / \mathrm{Eu}]_{r}^{\mathrm{L}}$, and $[\mathrm{Ag} / \mathrm{Eu}]_{r}^{\mathrm{H}}$ for scenario $\mathrm{A}$, and by $\log \epsilon_{\mathrm{L}}(\mathrm{Ag}), \log \epsilon_{\mathrm{L}}(\mathrm{Eu}), \log \epsilon_{\mathrm{H}}(\mathrm{Eu})$, and $[\mathrm{Ag} / \mathrm{Eu}]_{r}^{\mathrm{H}}$ for scenario B. From equations (33)-(35), we have

$$
\log \epsilon_{\mathrm{L}}(\mathrm{Ag})=\log \epsilon_{\odot, r}(\mathrm{Ag})+\log F_{r}^{\mathrm{L}}(\mathrm{Ag})-\log \left(T_{\mathrm{UP}} / \Delta_{\mathrm{L}}\right)
$$




$$
\begin{gathered}
\log \epsilon_{\mathrm{L}}(\mathrm{Eu})=\log \epsilon_{\odot, r}(\mathrm{Eu})+\log F_{r}^{\mathrm{L}}(\mathrm{Eu})-\log \left(T_{\mathrm{UP}} / \Delta_{\mathrm{L}}\right) \\
\log \epsilon_{\mathrm{H}}(\mathrm{Eu})=\log \epsilon_{\odot, r}(\mathrm{Eu})+\log F_{r}^{\mathrm{H}}(\mathrm{Eu})-\log \left(T_{\mathrm{UP}} / \Delta_{\mathrm{H}}\right) \\
{[\mathrm{Ag} / \mathrm{Eu}]_{r}^{\mathrm{L}}=\log \left[F_{r}^{\mathrm{L}}(\mathrm{Ag}) / F_{r}^{\mathrm{L}}(\mathrm{Eu})\right]}
\end{gathered}
$$

and

$$
[\mathrm{Ag} / \mathrm{Eu}]_{r}^{\mathrm{H}}=\log \left[F_{r}^{\mathrm{H}}(\mathrm{Ag}) / F_{r}^{\mathrm{H}}(\mathrm{Eu})\right]
$$

In the simplified model, we assume that $F_{r}^{\mathrm{L}}(\mathrm{Ag})=F_{r}^{\mathrm{L}}\left({ }^{127} \mathrm{I}\right)\left[\right.$ hence $\left.F_{r}^{\mathrm{H}}(\mathrm{Ag})=F_{r}^{\mathrm{H}}\left({ }^{127} \mathrm{I}\right)\right]$, and $F_{r}^{\mathrm{H}}(\mathrm{Eu})=F_{r}^{\mathrm{H}}\left({ }^{182} \mathrm{~W}\right)\left[\right.$ hence $\left.F_{r}^{\mathrm{L}}(\mathrm{Eu})=F_{r}^{\mathrm{L}}\left({ }^{182} \mathrm{~W}\right)\right]$.

In both scenarios $\mathrm{A}$ and $\mathrm{B}$, essentially all of the low mass nuclei are produced in the L events, i.e., $F_{r}^{\mathrm{L}}(\mathrm{Ag})=F_{r}^{\mathrm{L}}\left({ }^{127} \mathrm{I}\right) \approx 1$, and we have $\Delta_{\mathrm{L}}>\Delta_{\mathrm{L}}^{\min }=1.06 \times 10^{8}$ yr. Equation (A3) then gives

$$
\log \epsilon_{\mathrm{L}}(\mathrm{Ag}) \approx-0.81+\log \left(\frac{\Delta_{\mathrm{L}}}{10^{8} \mathrm{yr}}\right),
$$

where we have assumed $T_{\mathrm{UP}}=10^{10} \mathrm{yr}$ (here and elsewhere in the paper). This is the most general result of the model. For a reasonable range of $(1.06$ to 2$) \times 10^{8}$ yr for $\Delta_{\mathrm{L}}$, we obtain $\log \epsilon_{\mathrm{L}}(\mathrm{Ag}) \approx-0.78$ to -0.51 for both scenarios $\mathrm{A}$ and $\mathrm{B}$.

In scenario $\mathrm{A}$, we have $F_{r}^{\mathrm{H}}(\mathrm{Eu})=F_{r}^{\mathrm{H}}\left({ }^{182} \mathrm{~W}\right) \approx 0.45$ to 1 and $\Delta_{\mathrm{H}}<\Delta_{\mathrm{H}}^{\max }=1.85 \times 10^{7} \mathrm{yr}$. Equation (A5) then gives

$$
\log \epsilon_{\mathrm{H}}(\mathrm{Eu}) \approx(-2.83 \text { to }-2.49)+\log \left(\frac{\Delta_{\mathrm{H}}}{10^{7} \mathrm{yr}}\right) .
$$

For a reasonable range of $(1$ to 1.85$) \times 10^{7}$ yr for $\Delta_{\mathrm{H}}$, we obtain $\log \epsilon_{\mathrm{H}}(\mathrm{Eu}) \approx-2.83$ to -2.22. In addition, we have $F_{r}^{\mathrm{L}}(\mathrm{Ag})=F_{r}^{\mathrm{L}}\left({ }^{127} \mathrm{I}\right)>0.932, F_{r}^{\mathrm{H}}(\mathrm{Ag})=F_{r}^{\mathrm{H}}\left({ }^{127} \mathrm{I}\right)<0.068$, and $F_{r}^{\mathrm{L}}(\mathrm{Eu})=F_{r}^{\mathrm{L}}\left({ }^{182} \mathrm{~W}\right) \lesssim 0.55$. Equations $(\mathrm{A} 6)$ and $(\mathrm{A7})$ then give

$$
[\mathrm{Ag} / \mathrm{Eu}]_{r}^{\mathrm{L}}>0.23
$$

and

$$
[\mathrm{Ag} / \mathrm{Eu}]_{r}^{\mathrm{H}}<-0.82
$$


In scenario $\mathrm{B}$, we have $F_{r}^{\mathrm{L}}(\mathrm{Eu})=F_{r}^{\mathrm{L}}\left({ }^{182} \mathrm{~W}\right) \approx 0.55$ to 1 and $\Delta_{\mathrm{L}}>\Delta_{\mathrm{L}}^{\min }=1.06 \times 10^{8} \mathrm{yr}$. Equation (A4) then gives

$$
\log \epsilon_{\mathrm{L}}(\mathrm{Eu}) \approx(-1.75 \text { to }-1.49)+\log \left(\frac{\Delta_{\mathrm{L}}}{10^{8} \mathrm{yr}}\right) .
$$

For a reasonable range of $(1.06$ to 2$) \times 10^{8}$ yr for $\Delta_{\mathrm{L}}$, we obtain $\log \epsilon_{\mathrm{L}}(\mathrm{Eu}) \approx-1.72$ to -1.19 .

We note that although there is no restriction on $\Delta_{\mathrm{H}}$ in scenario $\mathrm{B}$, the yield of Eu in an $\mathrm{H}$ event is still well constrained by equation (29). With $F_{r}^{\mathrm{H}}(\mathrm{Eu})=F_{r}^{\mathrm{H}}\left({ }^{182} \mathrm{~W}\right)$, this equation can be rewritten as

$$
\frac{1}{F_{r}^{\mathrm{H}}(\mathrm{Eu})}\left(\frac{T_{\mathrm{UP}}}{\Delta_{\mathrm{H}}}\right) \approx\left(\frac{1}{C_{\mathrm{Hf}}}\right) \frac{T_{\mathrm{UP}} / \bar{\tau}_{182}}{1-\exp \left(-\Delta_{\mathrm{H}} / \bar{\tau}_{182}\right)},
$$

which gives

$$
\log \epsilon_{\mathrm{H}}(\mathrm{Eu}) \approx-2.72+\log \left[1-\exp \left(-\Delta_{\mathrm{H}} / \bar{\tau}_{182}\right)\right]
$$

For $\Delta_{\mathrm{H}} \gtrsim 10^{7} \mathrm{yr}$, we obtain $\log \epsilon_{\mathrm{H}}(\mathrm{Eu}) \approx-2.99$ to -2.72 . Similarly, equation (28) can be rewritten as

$$
\frac{1}{F_{r}^{\mathrm{H}}(\mathrm{Ag})}\left(\frac{T_{\mathrm{UP}}}{\Delta_{\mathrm{H}}}\right)>\left(\frac{1}{C_{\mathrm{I}}}\right) \frac{T_{\mathrm{UP}} / \bar{\tau}_{129}}{1-\exp \left(-\Delta_{\mathrm{H}} / \bar{\tau}_{129}\right)}
$$

which gives

$$
\log \epsilon_{\mathrm{H}}(\mathrm{Ag})<-2.81+\log \left[1-\exp \left(-\Delta_{\mathrm{H}} / \bar{\tau}_{129}\right)\right]
$$

Combining equations (A14) and (A16), we obtain $\log \epsilon_{\mathrm{H}}(\mathrm{Ag})-\log \epsilon_{\mathrm{H}}(\mathrm{Eu})<-0.09$, which corresponds to

$$
[\mathrm{Ag} / \mathrm{Eu}]_{r}^{\mathrm{H}}<-0.77 .
$$




\section{REFERENCES}

Anders, E., \& Grevesse, N. 1989, Geochim. Cosmochim. Acta, 53, 197

Arlandini, C., Käppeler, F., Wisshak, K., Gallino, R., Lugaro, M., Busso, M., \& Straniero, O. 1999, ApJ, in preparation

Brazzle, R. H., Pravdivtseva, O. V., Meshik, A. P., \& Hohenberg, C. M. 1999, Geochim. Cosmochim. Acta, in press

Brown, G. E., \& Bethe, H. A. 1994, ApJ, 423, 659

Busso, M., Gallino, R., \& Wasserburg, G. J. 1999, ARA\&A, in press

Cameron, A. G. W. 1993, in Protostars and Planets III, ed. E. H. Levy \& J. L. Lunine (Tucson: Univ. of Arizona Press), 47

Crawford, J. L., Sneden, C., King, J. R., Boesgaard, A. M., \& Deliyannis, C. P. 1998, AJ, 116,2489

Cowan, J. J., Burris, D. L., Sneden, C., McWilliam A., \& Preston, G. W. 1995, ApJ, 439, L51

Cowan, J. J., Sneden, C., Truran, J. W., \& Burris, D. L. 1996, ApJ, 460, L115

Cowan, J. J., McWilliam, A., Sneden, C., \& Burris, D. L. 1997, ApJ, 480, 246

Cowan, J. J., Pfeiffer, B., Kratz, K.-L., Thielemann, F.-K., Sneden, C., Burles, S., Tytler, D., \& Beers, T. C. 1999, ApJ, 521, in press

Cowan, J. J., \& Sneden, C. 1999, private communication

Fowler, W. A. 1961, in Proc. Rutherford Jubilee International Conf., ed. J. B. Birks (New York: Academic Press), 640

Fowler, W. A., \& Hoyle, F. 1960, Ann. Phys., 10, 280 
Freiburghaus, C., Rembges, J.-F., Rauscher, T., Kolbe, E., Thielemann, F.-K., Kratz, K.-L., Pfeiffer, B., \& Cowan, J. J. 1999, ApJ, in press

Harper, C. L., \& Jacobsen, S. B. 1996, Geochim. Cosmochim. Acta, 60, 1131

Hoffman, R. D., Woosley, S. E., \& Qian, Y.-Z. 1997, ApJ, 482, 951

Ishimaru, Y., \& Wanajo, S. 1999, ApJ, 511, L33

Jeffrey, P. M., \& Reynolds, J. H. 1961, J. Geophys. Res., 66, 3582

Käppeler, F., Beer, H., \& Wisshak, K. 1989, Rep. Prog. Phys., 52, 945

Käppeler, F., Jaag, S., Bao, Z. Y., \& Reffo, G. 1991, ApJ, 366, 605

Kratz, K.-L., Bitouzet, J.-P., Thielemann, F.-K., Möller, P., \& Pfeiffer, B. 1993, ApJ, 403, 216

Lee, D.-C., \& Halliday, A. N. 1995, Nature, 378, 771

Lee, D.-C., \& Halliday, A. N. 1996, Science, 274, 1876

Lee, D.-C., \& Halliday, A. N. 1997, Nature, 388, 854

Lee, D.-C., \& Halliday, A. N. 1999, Chem. Geol., in press

McWilliam A. 1997, ARA\&A, 35, 503

McWilliam A. 1998, AJ, 115, 1640

McWilliam A., Preston, G. W., Sneden, C., \& Searle, L. 1995, AJ, 109, 2757

McWilliam A., \& Searle, L. 1999, in Galaxy Evolution: Connecting the Distant Universe with the Local Fossil Record, ed. M. Spite (Dordrecht: Kluwer), in press

Meyer, B. S., \& Brown, J. S. 1997, ApJS, 112, 199

Meyer, B. S., Mathews, G. J., Howard, W. M., Woosley, S. E., \& Hoffman, R. D. 1992, ApJ, 399, 656

Qian, Y.-Z., Vogel, P., \& Wasserburg, G. J. 1998, ApJ, 494, 285 (QVW98) 
Qian, Y.-Z., Vogel, P., \& Wasserburg, G. J. 1999, ApJ, 524, in press

Qian, Y.-Z., \& Woosley, S. E. 1996, ApJ, 471, 331

Raiteri, C. M., Villata, M., Gallino, R., Busso, M., \& Cravanzola, A. 1999, ApJ, 518, in press

Reynolds, J. H. 1960, Phys. Rev. Lett., 4, 8

Schramm, D. N., \& Wasserburg, G. J. 1970, ApJ, 162, 57

Sneden, C., Cowan, J. J., Burris, D. L., \& Truran, J. W. 1998, ApJ, 496, 235

Sneden, C., McWilliam, A., Preston, G. W., Cowan, J. J., Burris, D. L., \& Armosky, B. J. 1996, ApJ, 467, 819

Sneden, C., Preston, G. W., McWilliam, A., \& Leonard, S. 1994, ApJ, 431, L27

Sneden, C., et al. 1999, in preparation

Takahashi, K., Witti, J., \& Janka, H.-T. 1994, A\&A, 286, 857

Timmes, F. X., Woosley, S. E., \& Weaver, T. A. 1995, ApJS, 98, 617

Travaglio, C., Galli, D., Gallino, R., Busso, M., Ferrini, F., \& Straniero, O. 1999, ApJ, in press

Tsujimoto, T., \& Shigeyama, T. 1998, ApJ, 508, L151

Wasserburg, G. J., Busso, M., \& Gallino, R. 1996, ApJ, 466, L109 (WBG96)

Wasserburg, G. J., Busso, M., Gallino, R., \& Raiteri, C. M. 1994, ApJ, 424, 412

Witti, J., Janka, H.-T., \& Takahashi, K. 1994, A\&A, 286, 841

Woosley, S. E., Wilson, J. R., Mathews, G. J., Hoffman, R. D., \& Meyer, B. S. 1994, ApJ, 433, 229 
Fig. 1.- Typical characteristics of the $\mathrm{H}$ and $\mathrm{L}$ events in scenario A. With the assumption of $\Delta_{\mathrm{H}} / \Delta_{\mathrm{L}}=0.1$ in this example, the restrictions $\Delta_{\mathrm{H}}<\Delta_{\mathrm{H}}^{\max }=1.85 \times 10^{7} \mathrm{yr}$ and $\Delta_{\mathrm{L}}>\Delta_{\mathrm{L}}^{\min }=1.06 \times 10^{8} \mathrm{yr}$ specify a physical range of $(1.06$ to 1.85$) \times 10^{8}$ yr for $\Delta_{\mathrm{L}}$. (a) The relative yield of ${ }^{127} \mathrm{I}$ to ${ }^{182} \mathrm{~W}$ in an $\mathrm{H}$ or $\mathrm{L}$ event with respect to the corresponding solar $r$-process abundance ratio, measured by $U_{\mathrm{H}}$ or $U_{\mathrm{L}}$ (cf. eqs. [20] and [23]), respectively, as a function of $\Delta_{\mathrm{L}}$. (b) The fraction of ${ }^{127} \mathrm{I}$ contributed by the L events to the total solar $r$-process abundance of ${ }^{127} \mathrm{I}, F_{r}^{\mathrm{L}}\left({ }^{127} \mathrm{I}\right)$ (cf. eq. 18 ), and the fraction of ${ }^{182} \mathrm{~W}$ contributed by the $\mathrm{H}$ events to the total solar $r$-process abundance of ${ }^{182} \mathrm{~W}, F_{r}^{\mathrm{H}}\left({ }^{182} \mathrm{~W}\right)$ (cf. eq. [19]), as functions of $\Delta_{\mathrm{L}}$. Note that $F_{r}^{\mathrm{L}}\left({ }^{127} \mathrm{I}\right)=1$ and $F_{r}^{\mathrm{H}}\left({ }^{182} \mathrm{~W}\right)=1$ correspond to the limiting cases of $\Delta_{\mathrm{L}}=\Delta_{\mathrm{L}}^{\min }$ and $\Delta_{\mathrm{H}}=\Delta_{\mathrm{H}}^{\max }$ (see $\S 2.1 .1$ ), respectively.

Fig. 2.- Typical characteristics of the $\mathrm{H}$ and $\mathrm{L}$ events in scenario B. As in Figure 1, $\Delta_{\mathrm{H}} / \Delta_{\mathrm{L}}=0.1$ is assumed. In addition, $\Delta_{\mathrm{L}} \leq 2 \times 10^{8} \mathrm{yr}$ is imposed in this example. (a) See caption for Figure $1(\mathrm{a})$. Note that $U_{\mathrm{L}}=1.44$ to 1.26 over the range of $(1.06$ to 2$) \times 10^{8} \mathrm{yr}$ for $\Delta_{\mathrm{L}}$, i.e., the relative yields in the low and high mass regions in an L event essentially follow the solar $r$-pattern. (b) See caption for Figure 1(b). As the L events dominate the production of both ${ }^{127} \mathrm{I}$ and ${ }^{182} \mathrm{~W}, F_{r}^{\mathrm{L}}\left({ }^{127} \mathrm{I}\right)$ and $F_{r}^{\mathrm{L}}\left({ }^{182} \mathrm{~W}\right)=1-F_{r}^{\mathrm{H}}\left({ }^{182} \mathrm{~W}\right)$ are shown as functions of $\Delta_{\mathrm{L}}$. Note that $F_{r}^{\mathrm{L}}\left({ }^{127} \mathrm{I}\right)=1$ corresponds to the limiting case of $\Delta_{\mathrm{L}}=\Delta_{\mathrm{L}}^{\min }$ as in Figure 1.

Fig. 3.- (a) The yields of the stable $(\mathcal{S})$ nuclei ${ }^{127} \mathrm{I}$ and ${ }^{182} \mathrm{~W}$ in an $\mathrm{H}$ or $\mathrm{L}$ event shown in terms of the parameters $\delta \log \epsilon_{\mathrm{H}}(\mathcal{S}) \equiv \log \epsilon_{\mathrm{H}}(\mathcal{S})-\log \epsilon_{\odot, r}(\mathcal{S})$ or $\delta \log \epsilon_{\mathrm{L}}(\mathcal{S}) \equiv$ $\log \epsilon_{\mathrm{L}}(\mathcal{S})-\log \epsilon_{\odot, r}(\mathcal{S})$ as functions of $\Delta_{\mathrm{L}}$ for scenario A. These parameters are calculated from the values of $F_{r}^{\mathrm{L}}\left({ }^{127} \mathrm{I}\right)$ and $F_{r}^{\mathrm{H}}\left({ }^{182} \mathrm{~W}\right)$ shown in Figure $1(\mathrm{~b})$ with the use of equations (33) and (34). Note that $\log \epsilon_{\mathrm{H}}(\mathcal{S})$ or $\log \epsilon_{\mathrm{L}}(\mathcal{S})$ gives the abundance of $\mathcal{S}$ nuclei with respect to hydrogen in a star formed from an ISM contaminated by a single H or L event, respectively. In standard spectroscopic notation, $\log \epsilon(\mathcal{S})=\log (\mathcal{S} / \mathcal{H})+12$, where $\mathcal{S} / \mathcal{H}$ is the abundance 
ratio of the nuclide $\mathcal{S}$ to hydrogen in the star. (b) Same as (a), but for scenario B. The values of $F_{r}^{\mathrm{L}}\left({ }^{127} \mathrm{I}\right)$ and $F_{r}^{\mathrm{L}}\left({ }^{182} \mathrm{~W}\right)$ shown in Figure $2(\mathrm{~b})$ are used in the calculation.

Fig. 4.- (a) The solar $r$-process nuclear abundances (Käppeler et al. 1989, 1991) shown in terms of $\log \epsilon_{\odot, r}(A)$. The vertical dashed line indicates the boundary $(A=130)$ between the low and high mass regions chosen in our simplified two-component $r$-process model. (b) The solar $r$-process elemental abundances in the low and high mass regions shown in terms of $\log \epsilon_{\odot, r}(Z)$. As in (a), the vertical dashed line indicates the boundary between the two mass regions. This line is put at $Z=54(\mathrm{Xe})$ as $\mathrm{Xe}$ has $r$-process isotopes in both mass regions.

Fig. 5.- (a) The yields of $\mathrm{Ag}$ and $\mathrm{Eu}$ in an $\mathrm{H}$ or L event in scenario A shown in terms of $\log \epsilon_{\mathrm{H}}(\mathrm{Ag})$ and $\log \epsilon_{\mathrm{H}}(\mathrm{Eu})$ or $\log \epsilon_{\mathrm{L}}(\mathrm{Ag})$ and $\log \epsilon_{\mathrm{L}}(\mathrm{Eu})$ as functions of $\Delta_{\mathrm{L}}$. These $\log \epsilon$ values correspond to the abundances of $\mathrm{Ag}$ and $\mathrm{Eu}$ that should be observed in a star formed from an ISM contaminated by a single $\mathrm{H}$ or L event in scenario A. They are obtained from the solar $r$-process elemental abundances shown in Figure 4(b) with the use of the $\delta \log \epsilon_{\mathrm{H}}(\mathcal{S})$ and $\delta \log \epsilon_{\mathrm{L}}(\mathcal{S})$ values for ${ }^{127} \mathrm{I}$ and ${ }^{182} \mathrm{~W}$ shown in Figure $3(\mathrm{a})$. Note that in our simplified two-component $r$-process model, we assume, for example, $\delta \log \epsilon_{\mathrm{L}}(\mathrm{Ag})=\delta \log \epsilon_{\mathrm{L}}\left({ }^{127} \mathrm{I}\right)$ and $\delta \log \epsilon_{\mathrm{H}}(\mathrm{Eu})=\delta \log \epsilon_{\mathrm{H}}\left({ }^{182} \mathrm{~W}\right)$. (b) Same as (a), but for scenario B. The parameters shown in Figure 3(b) are used to obtain these results.

Fig. 6.- (a) The elemental yields in an $\mathrm{H}$ event in scenario A [solid curve labelled "H (A)"] shown in terms of $\log \epsilon(Z)$ for the case of $\Delta_{\mathrm{L}}=1.5 \times 10^{8} \mathrm{yr}$ and $\Delta_{\mathrm{H}} / \Delta_{\mathrm{L}}=0.1$. The dotted curve labelled "TS" is the solar $r$-pattern translated to match the yield of Eu (hence those of $Z>54$ by the assumption of our model). The vertical line at Xe $(Z=54)$ indicates the boundary between the low and high mass regions chosen in our model. With respect to the translated solar $r$-pattern, the yields below Xe are lower by 1.2 dex. (b) The elemental yields in an L event in scenario A [solid curve labelled "L (A)"] shown in terms of $\log \epsilon(Z)$ for the same case as in (a). Common symbols have the same meanings as in (a). With respect to 
the translated solar $r$-pattern, the yields below Xe are higher by 0.8 dex.

Fig. 7.- (a) The elemental yields in an $\mathrm{H}$ event in scenario B [solid curve labelled "H (B)"] shown in terms of $\log \epsilon(Z)$ for the case of $\Delta_{\mathrm{L}}=1.5 \times 10^{8} \mathrm{yr}$ and $\Delta_{\mathrm{H}} / \Delta_{\mathrm{L}}=0.1$. Common symbols have the same meanings as in Figure 6. With respect to the translated solar $r$ pattern, the yields below Xe are lower by 1.0 dex. (b) The elemental yields in an L event in scenario B [solid curve labelled "L (B)"] shown in terms of $\log \epsilon(Z)$ for the same case as in (a). Common symbols have the same meanings as in Figure 6. The yields in both the low and high mass regions essentially coincide with the translated solar $r$-pattern.

Fig. 8.- (a) Comparison of the yields in an H or L event in scenario A with the observed abundances in HD 122563 given by Sneden et al. (1998). The filled squares with error bars represent measured abundances while the open triangles indicate upper limits. The dotted curve labelled "TS" is the solar $r$-pattern translated to match the observed Eu abundance (indicated by "Eu" below the filled square). In contrast, the solid curve labelled "H (A)" or the dashed curve labelled "L (A)" shows the yields in an $\mathrm{H}$ or L event in scenario A [cf. Figs. $6(\mathrm{a})$ or (b)] obtained directly from our model (i.e., no fitting is attempted). A decisive test of the agreement between the model and the data is the abundances of elements such as Ag in the low mass region, the observations of which are under way (Sneden et al. 1999). (b) Comparison of the yields in an L event in scenario B with the observed abundances in HD 115444 given by Sneden et al. (1998). Data symbols are the same as in (a). The solid curve labelled "L (B)" shows the yields in an L event in scenario B [cf. Fig. 7(b)] obtained directly from our model. The solar $r$-pattern translated to match the observed Eu abundance (not shown) is almost identical to the solid curve. As in (a), the abundances in the low mass region being observed by Sneden et al. (1999) will test decisively the agreement between the model and the data.

Fig. 9.- The observed Eu abundances and Ba/Eu abundance ratios for 15 very metal-poor 
stars shown against the corresponding Fe abundances (or metallicities $[\mathrm{Fe} / \mathrm{H}]$ ). The open squares represent data from McWilliam et al. (1995) and McWilliam (1998), while the filled triangles represent data from Sneden et al. $(1996,1998,1999)$. The quantity $[\mathrm{Ba} / \mathrm{Eu}]_{r}$ is defined as $[\mathrm{Ba} / \mathrm{Eu}]_{r} \equiv \log (\mathrm{Ba} / \mathrm{Eu})-\log \left[N_{\odot, r}(\mathrm{Ba}) / N_{\odot, r}(\mathrm{Eu})\right]$, and measures the deviation of the $\mathrm{Ba} / \mathrm{Eu}$ abundance ratio from the corresponding solar $r$-process value. Note that except for one star, all the other stars with $[\mathrm{Fe} / \mathrm{H}] \leq-2.41$ shown in this figure have $[\mathrm{Ba} / \mathrm{Eu}]_{r} \approx 0$ to 0.3 . In contrast, the Eu abundances vary by about 1.5 dex for the same group of stars.

Fig. 10.- (a) Comparison of the result from a mixture of multiple $r$-process events with the observed abundances in HD 115444 given by Sneden et al. (1998). Common symbols have the same meanings as in Figure 8. The number of pure $\mathrm{H}$ events in scenario A [cf. Fig. 6(a)] required to account for the observed Eu abundance is seven. The abundances from such a mixture are shown by the solid curve labelled "7 H (A)," which is far below the translated solar $r$-pattern in the low mass region. The result from a mixture of seven $\mathrm{H}$ events and one L event [cf. Fig. 6(b)] in scenario A (not shown) essentially coincides with the translated solar $r$-pattern. Observations of abundances in the low mass region will provide a crucial test for the viability of using mixtures of multiple $r$-process events to explain the data in HD 115444. (b) Comparison of results from mixtures of multiple $r$-process events with the observed abundances in CS 22892-052 given by Sneden et al. (1996, 1999). Common symbols have the same meanings as in Figure 8. The number of pure $\mathrm{H}$ events in scenario A [cf. Fig. 6(a)] required to account for the observed Eu abundance is 27. The abundances from such a mixture are shown by the dashed curve labelled " $27 \mathrm{H} \mathrm{(A),"} \mathrm{which} \mathrm{is} \mathrm{far} \mathrm{below}$ the translated solar $r$-pattern in the low mass region. This mixture cannot account for the preliminary value of the observed Ag abundance (Cowan \& Sneden 1999) indicated by the open square. However, the result from a mixture of $26 \mathrm{H}$ events and one L event [cf. Fig. 6(b)] in scenario A, shown as the solid curve labelled "26 H (A) + $1 \mathrm{~L}(\mathrm{~A})$," matches both the preliminary Ag data and the observed abundances in the high mass region. 
Table 1. Input Data for the Two-Component $r$-Process Model

\begin{tabular}{lll}
\hline \hline \multicolumn{1}{c}{${ }^{129} \mathrm{I}$} & \multicolumn{1}{c}{${ }^{182} \mathrm{Hf}$} & Note \\
\hline $\bar{\tau}_{129}=2.27 \times 10^{7} \mathrm{yr}$ & $\bar{\tau}_{182}=1.30 \times 10^{7} \mathrm{yr}$ & (a) \\
$\left({ }^{129} \mathrm{I} /{ }^{127} \mathrm{I}\right) \mathrm{SSF}=10^{-4}$ & $\left({ }^{182} \mathrm{Hf} /{ }^{180} \mathrm{Hf}\right)_{\mathrm{SSF}}=2.4 \times 10^{-4}$ & (b) \\
$N_{\odot}\left({ }^{127} \mathrm{I}\right)=0.90$ & $N_{\odot}\left({ }^{180} \mathrm{Hf}\right)=0.0541$ & (c) \\
$N_{\odot, r}\left({ }^{127} \mathrm{I}\right) \approx N_{\odot}\left({ }^{127} \mathrm{I}\right)$ & $N_{\odot, r}\left({ }^{182} \mathrm{~W}\right)=0.0222$ & (d) \\
$C_{\mathrm{I}}=0.0441$ & $C_{\mathrm{Hf}}=0.450$ & (e) \\
\hline
\end{tabular}

${ }^{\text {(a) }}$ Lifetimes of ${ }^{129} \mathrm{I}$ and ${ }^{182} \mathrm{Hf}$.

(b) Meteoritic data on ${ }^{129}$ I (Reynolds 1960; Jeffrey \& Reynolds 1961; Brazzle et al. 1999) and ${ }^{182}$ Hf (Harper \& Jacobsen 1996; Lee \& Halliday 1995, 1996, 1997, 1999).

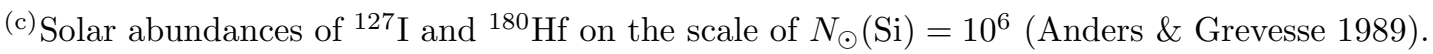

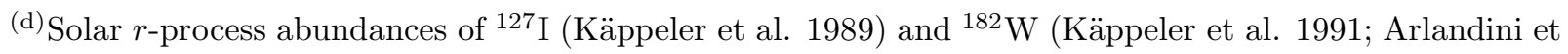
al. 1999) on the scale of $N_{\odot}(\mathrm{Si})=10^{6}$.

${ }^{(\mathrm{e})}$ Defined in eqs. (11) and (12) and evaluated for $Y_{129} / Y_{127}=1\left(C_{\mathrm{I}}\right)$ and $T_{\mathrm{UP}}=10^{10} \mathrm{yr}\left(C_{\mathrm{I}}\right.$ and $\left.C_{\mathrm{Hf}}\right)$. 
Table 2. Some Solar $r$-Process Elemental Abundance Data

\begin{tabular}{lllll}
\hline \hline Element & $Z^{(\mathrm{a})}$ & \multicolumn{1}{c}{$A^{(\mathrm{b})}$} & $N_{\odot, r}{ }^{(\mathrm{c})}$ & $\log \epsilon_{\odot, r}{ }^{(\mathrm{d})}$ \\
\hline $\mathrm{Pd}$ & 46 & $105,106,108,110$ & 0.770 & 1.44 \\
$\mathrm{Ag}$ & 47 & 107,109 & 0.435 & 1.19 \\
$\mathrm{Cd}$ & 48 & $111-114,116$ & 0.771 & 1.44 \\
$\mathrm{Ba}$ & 56 & $135,137,138$ & $0.726^{(\mathrm{e})}$ & 1.42 \\
$\mathrm{Eu}$ & 63 & 151,153 & 0.0907 & 0.51 \\
\hline
\end{tabular}

(a) Atomic number.

(b) Mass numbers of $r$-process isotopes.

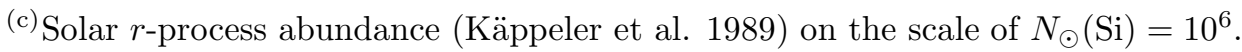

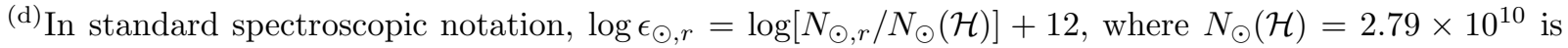
the solar abundance of hydrogen (Anders \& Grevesse 1989) on the scale of $N_{\odot}(\mathrm{Si})=10^{6}$.

${ }^{\text {(e) }}$ Assumed to be $3 \times N_{\odot, r}\left({ }^{135} \mathrm{Ba}\right)$ in view of the uncertainties in the $s$-process abundances (cf. Käppeler et al. 1989; Arlandini et al. 1999). 
Table 3. Typical Yields in the Simplified Two-Component $r$-Process Model

\begin{tabular}{lllllll}
\hline \hline Scenario $^{(\mathrm{a})}$ & $\log \epsilon_{\mathrm{H}}(\mathrm{Ag})^{(\mathrm{b})}$ & $\log \epsilon_{\mathrm{H}}(\mathrm{Eu})^{(\mathrm{b})}$ & {$[\mathrm{Ag} / \mathrm{Eu}]_{r}^{\mathrm{H}(\mathrm{c})}$} & $\log \epsilon_{\mathrm{L}}(\mathrm{Ag})^{(\mathrm{b})}$ & $\log \epsilon_{\mathrm{L}}(\mathrm{Eu})^{(\mathrm{b})}$ & {$[\mathrm{Ag} / \mathrm{Eu}]_{r}^{\mathrm{L}(\mathrm{c})}$} \\
\hline \multirow{2}{*}{$\mathrm{A}$} & -2.93 & -2.38 & -1.22 & -0.65 & -2.13 & 0.80 \\
$\mathrm{~B}$ & -3.22 & -2.89 & -1.01 & -0.64 & -1.45 & 0.12 \\
\hline
\end{tabular}

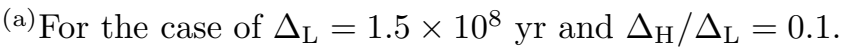

(b) The yields of $\mathrm{Ag}$ and $\mathrm{Eu}$ in an $\mathrm{H}$ or $\mathrm{L}$ event in terms of the $\log \epsilon$ values for a star formed from an ISM contaminated by an $\mathrm{H}$ or $\mathrm{L}$ event. In standard spectroscopic notation, $\log \epsilon(\mathrm{Ag})=\log (\mathrm{Ag} / \mathcal{H})+12$, where $\mathrm{Ag} / \mathcal{H}$ is the abundance ratio of $\mathrm{Ag}$ to hydrogen in the star.

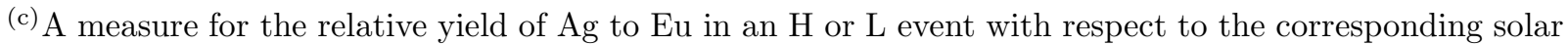
$r$-process abundance ratio. See eq. (35) for definition. 
Table 4. Comparison with Observational Data on Very Metal-Poor Stars

\begin{tabular}{llllll}
\hline \hline \multicolumn{1}{c}{ Star $^{(\mathrm{a})}$} & {$[\mathrm{Fe} / \mathrm{H}]^{(\mathrm{b})}$} & $\log \epsilon(\mathrm{Fe})^{(\mathrm{c})}$ & $\log \epsilon(\mathrm{Eu})^{(\mathrm{d})}$ & Single H Event?(e) $^{(\mathrm{e})}$ & Single L Event?(e) \\
\hline HD 122563 & -2.71 & 4.8 & -2.48 & A (yes), B (?) & A (yes) \\
HD 115444 & -2.77 & 4.7 & -1.53 & no & B (yes) \\
CS 22892-052 & -3.1 & 4.4 & -0.94 & no & no \\
HD 126238 & -1.67 & 5.8 & -0.92 & no & no \\
\hline
\end{tabular}

(a) Data on CS 22892-052 from Sneden et al. (1996, 1999), and the rest from Sneden et al. (1998).

(b) Metallicity.

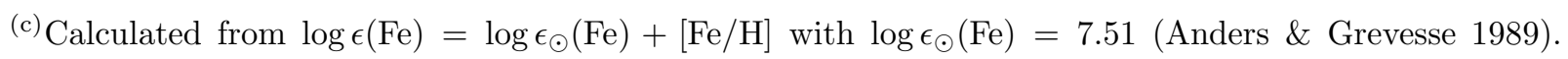
Note that for uniform Fe production by Type II supernovae, the yield in an H or L event corresponds to $\log \epsilon(\mathrm{Fe}) \approx 4.2$.

(d) In standard spectroscopic notation, $\log \epsilon(\mathrm{Eu})=\log (\mathrm{Eu} / \mathcal{H})+12$, where $\mathrm{Eu} / \mathcal{H}$ is the abundance ratio of Eu to hydrogen in the star.

(e) "A" and "B" stand for scenarios A and B, respectively. A question mark (?) indicates a marginal case. Note that for both scenarios $\mathrm{A}$ and $\mathrm{B}$, the expected range of Eu yield corresponds to $\log \epsilon(\mathrm{Eu})=-2.98$ to -2.22 for an $\mathrm{H}$ event and $\log \epsilon(\mathrm{Eu}) \approx-2.4$ to -1.3 for an $\mathrm{L}$ event. See Table 3 for typical Eu yields. 


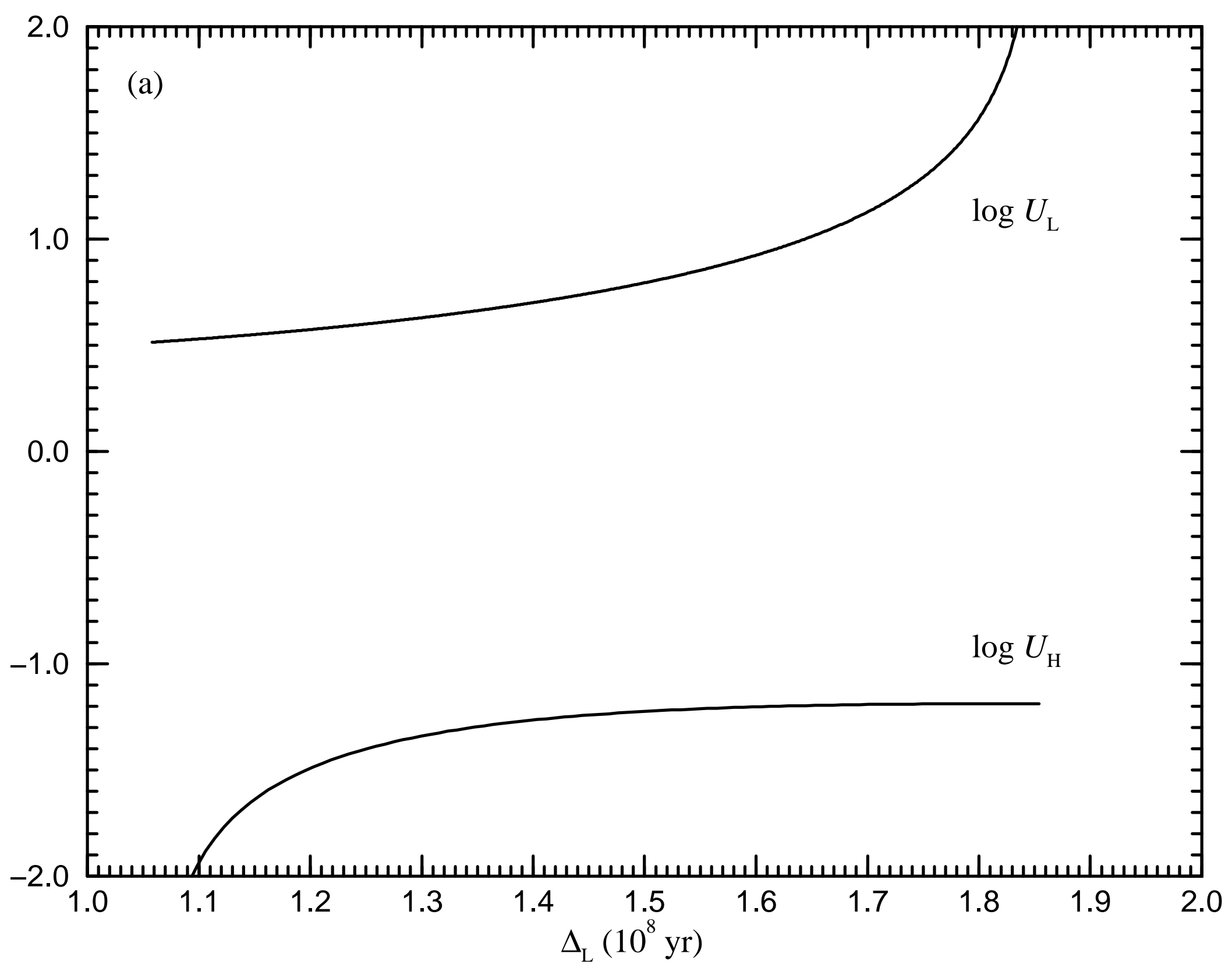




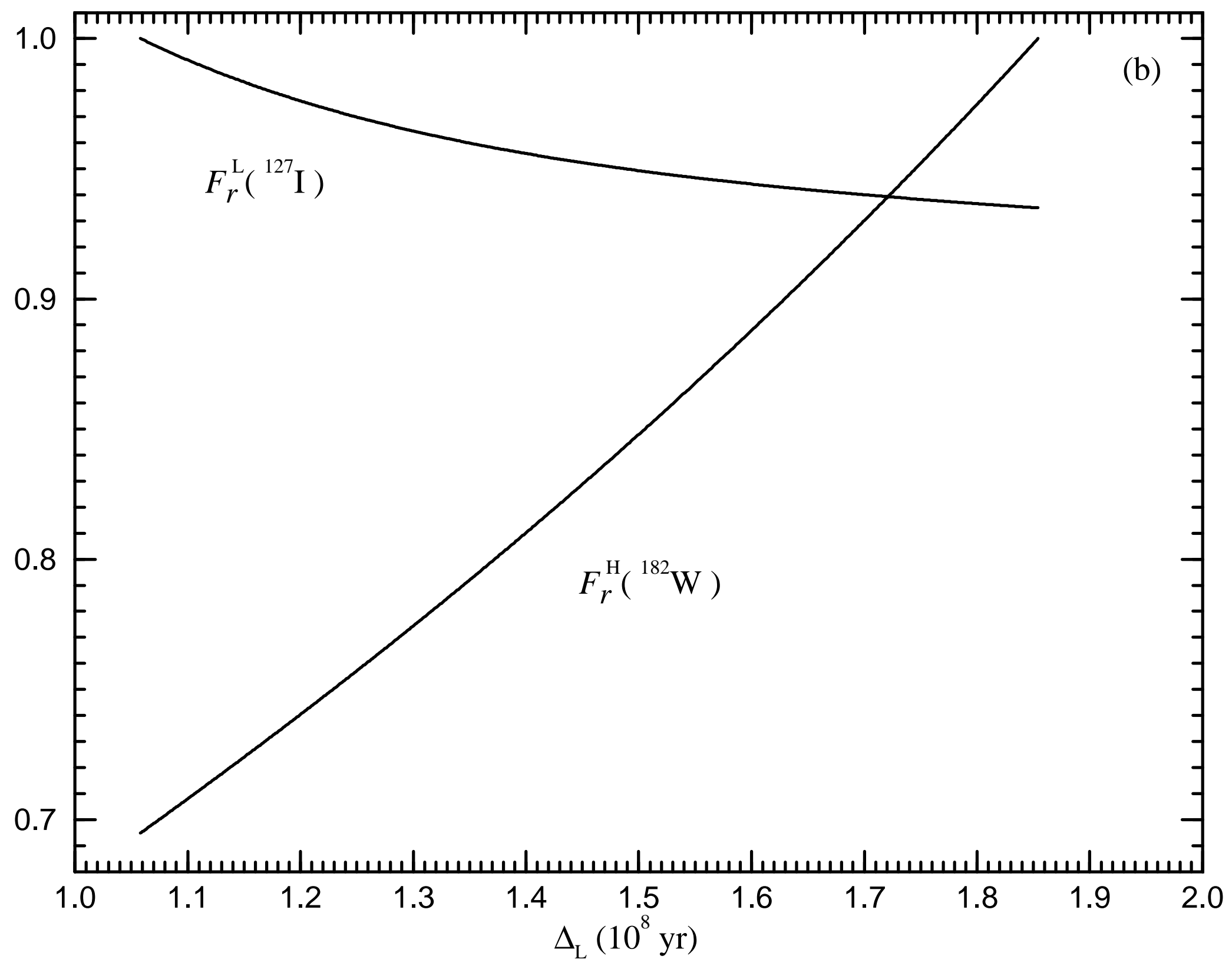




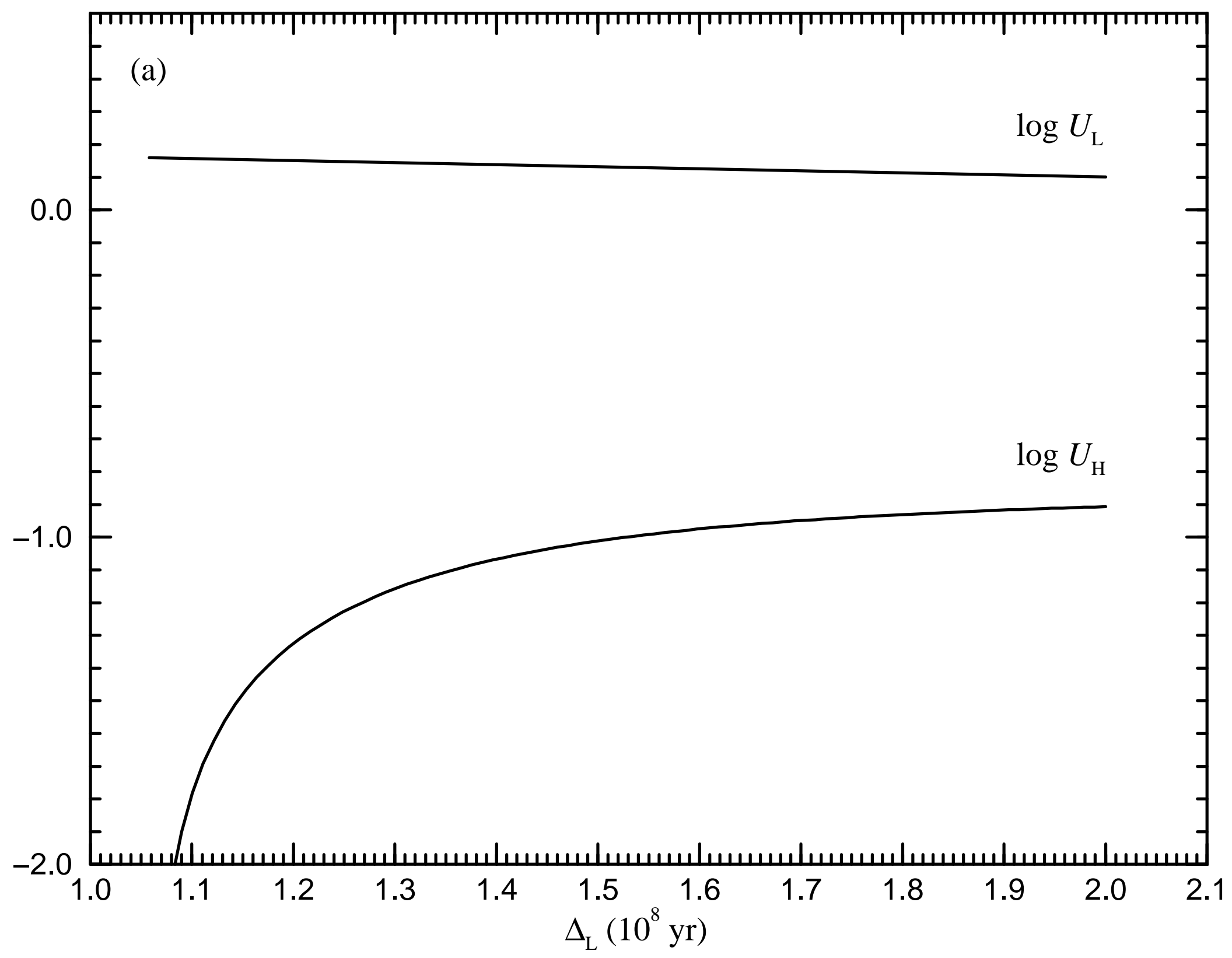




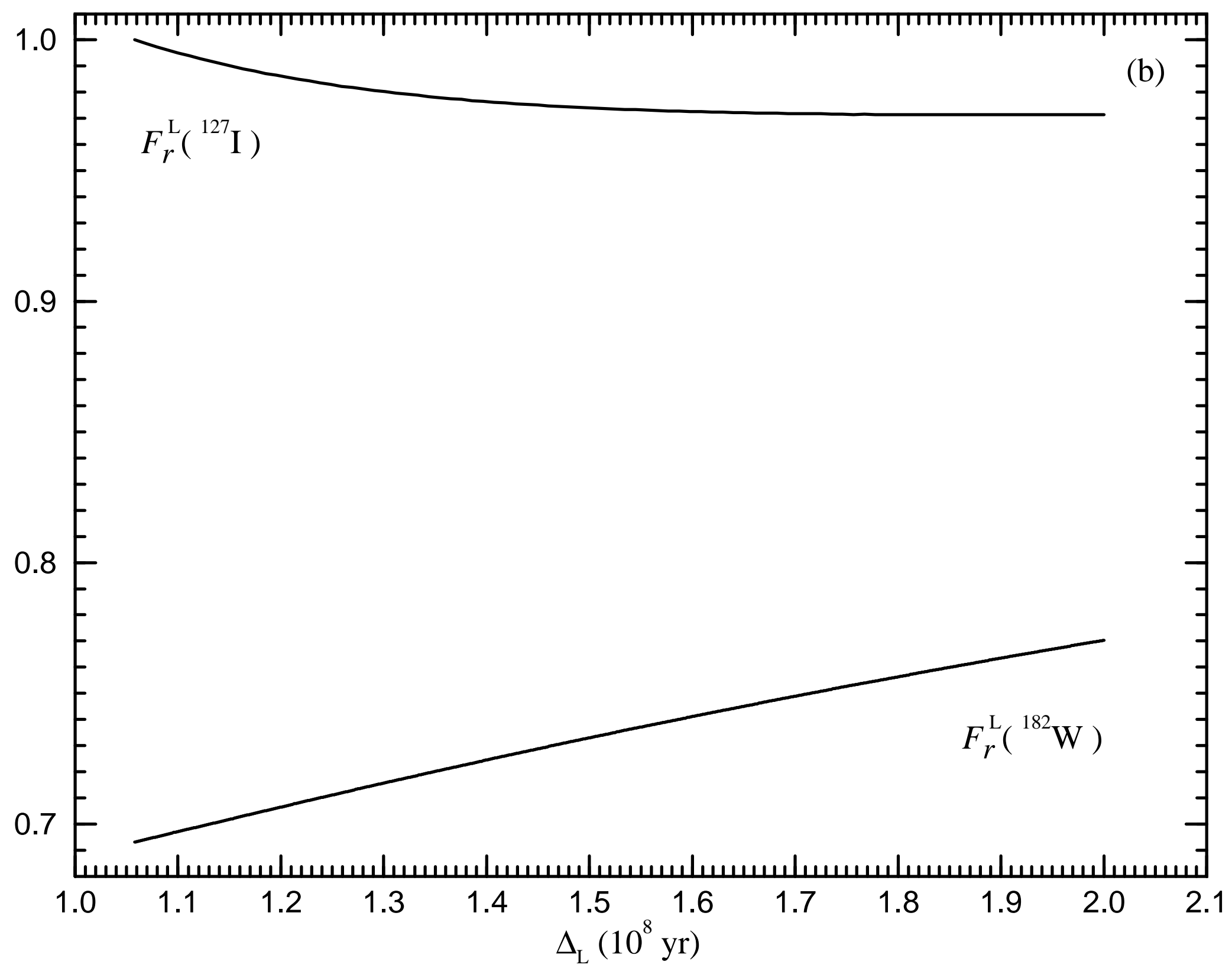




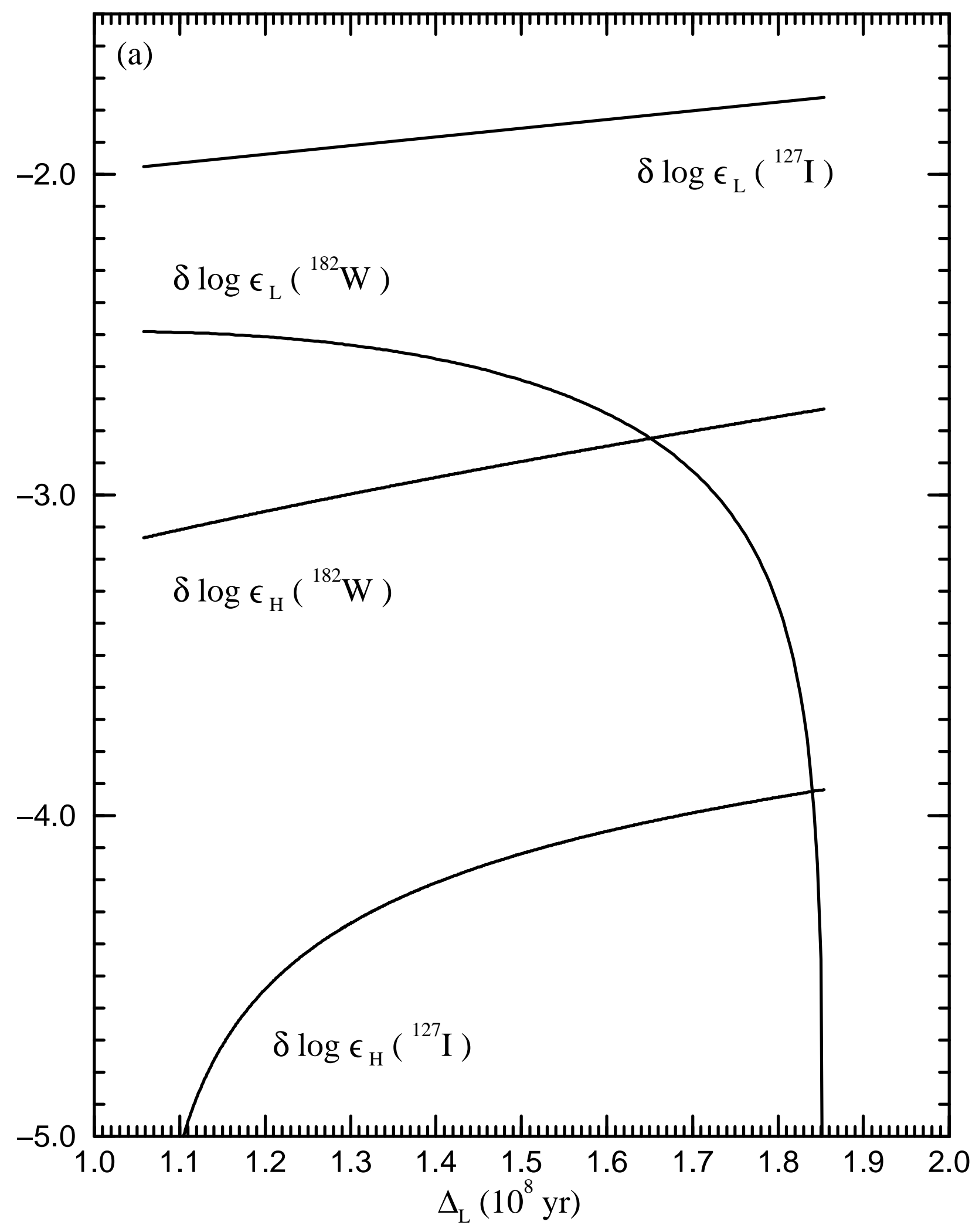




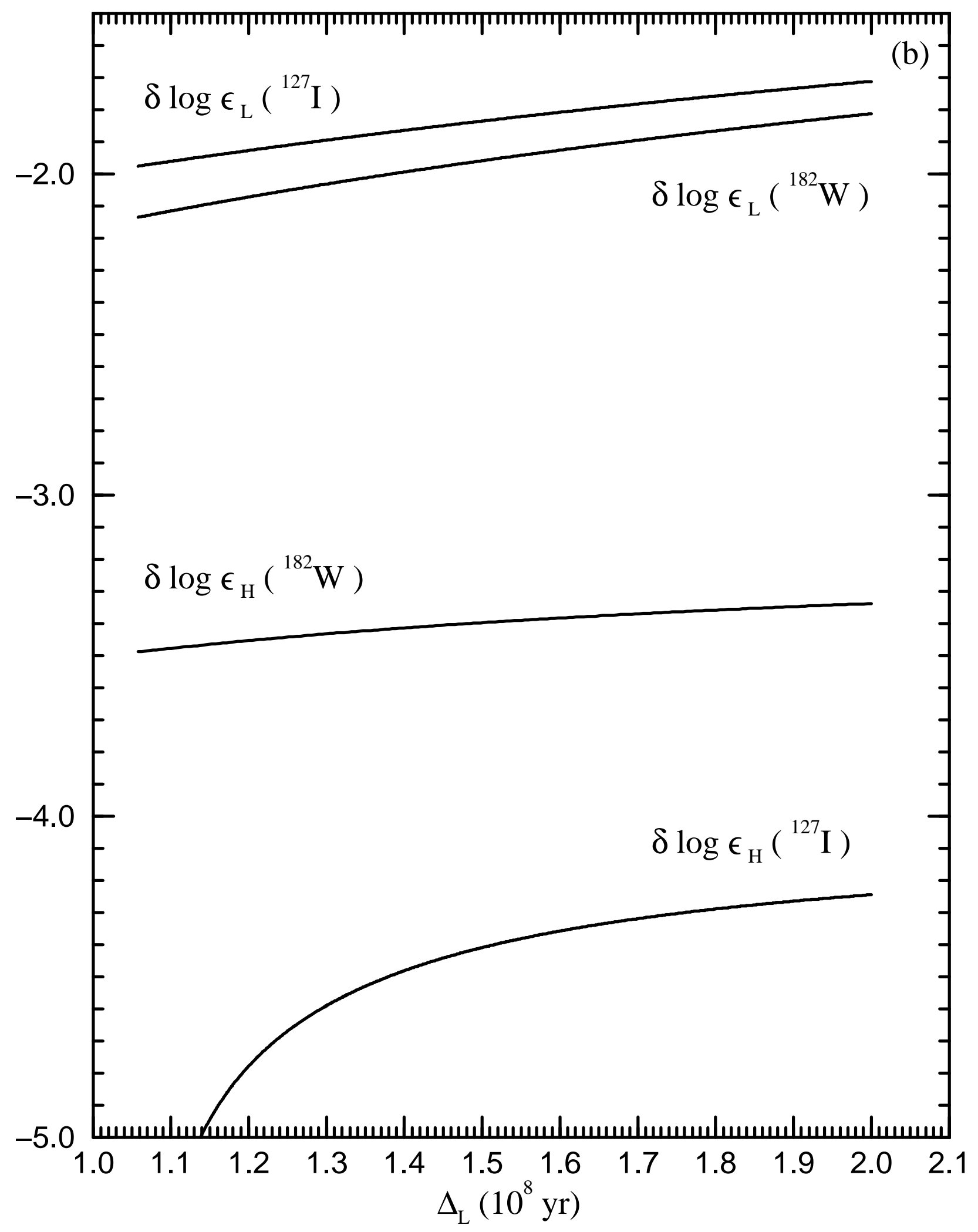




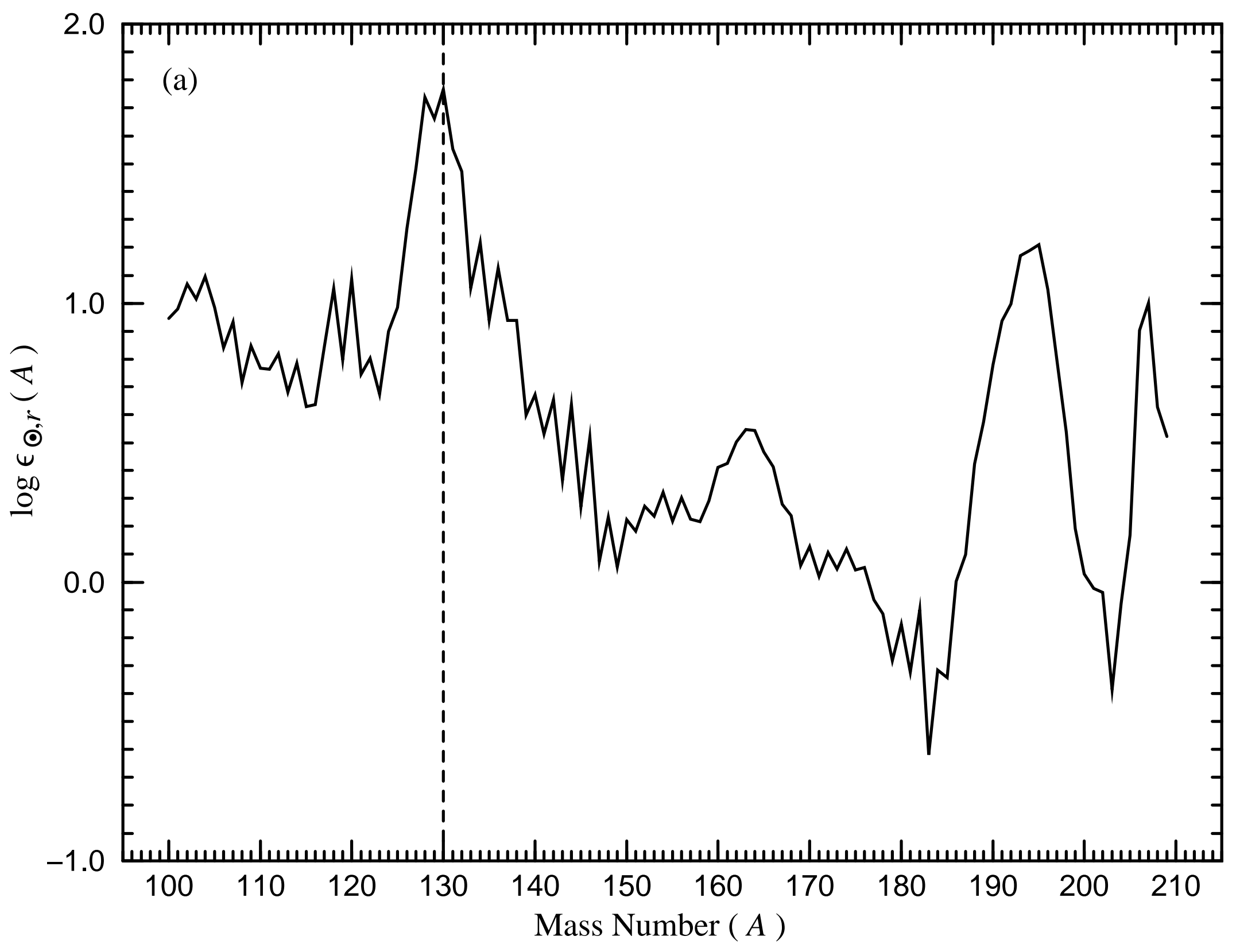




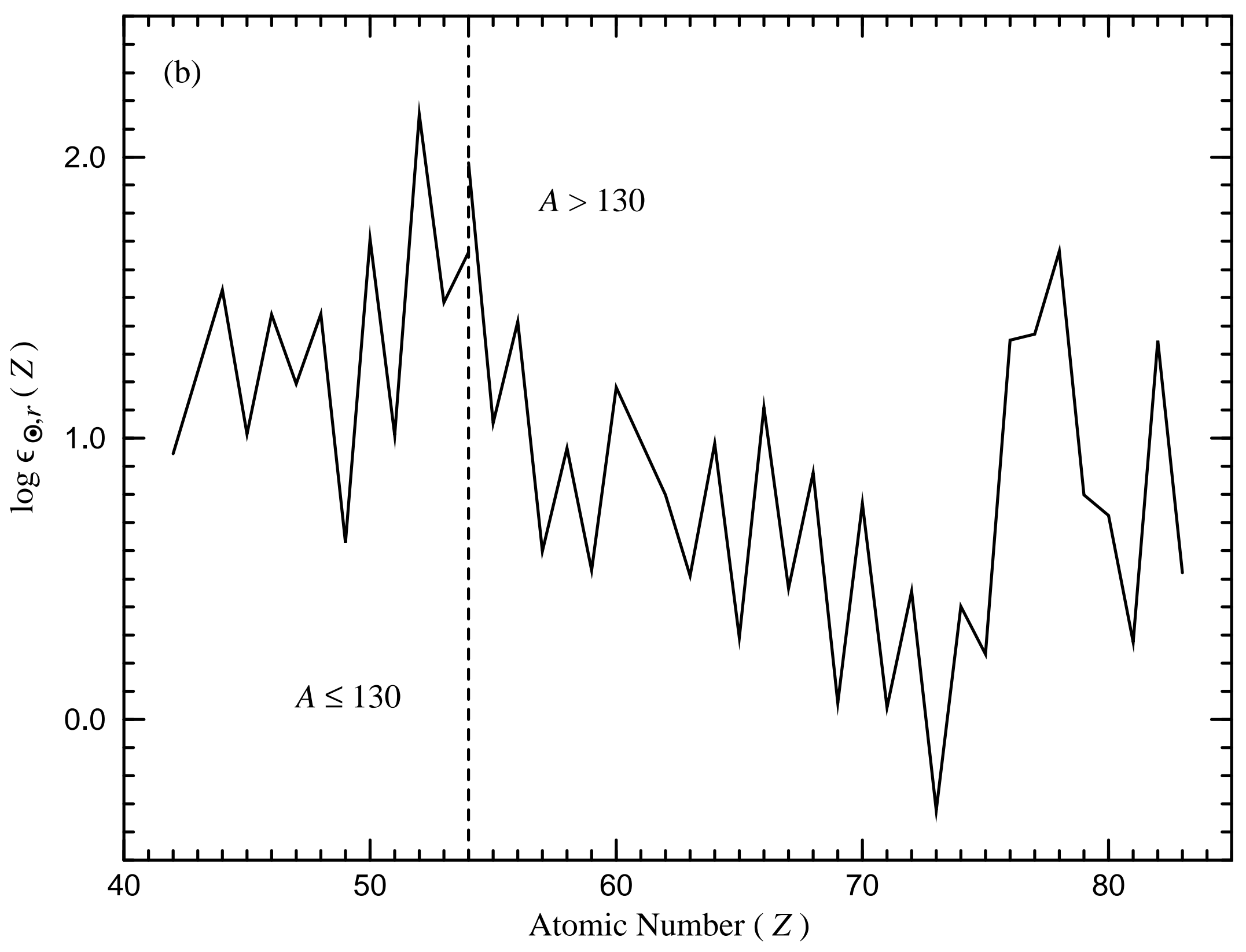




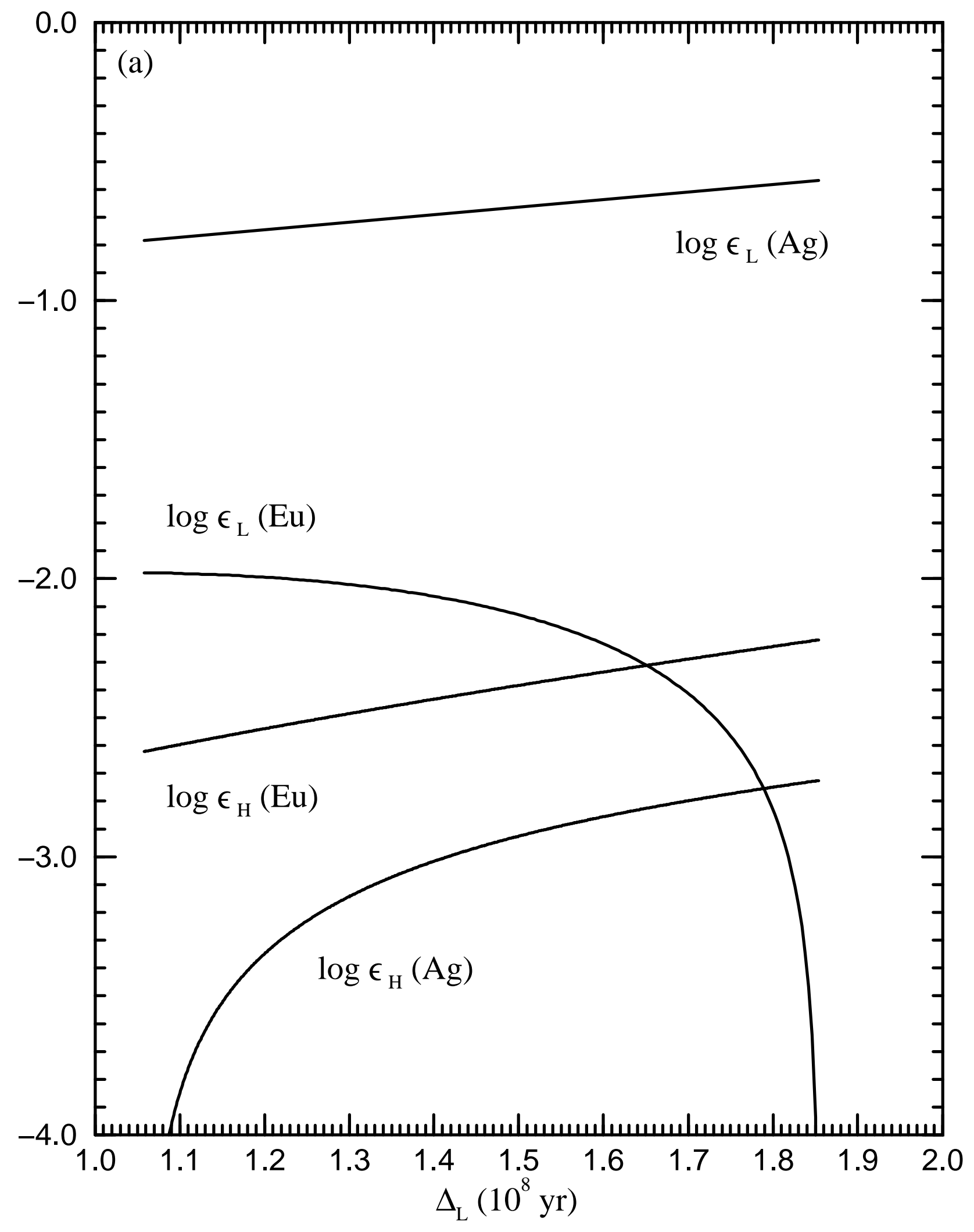




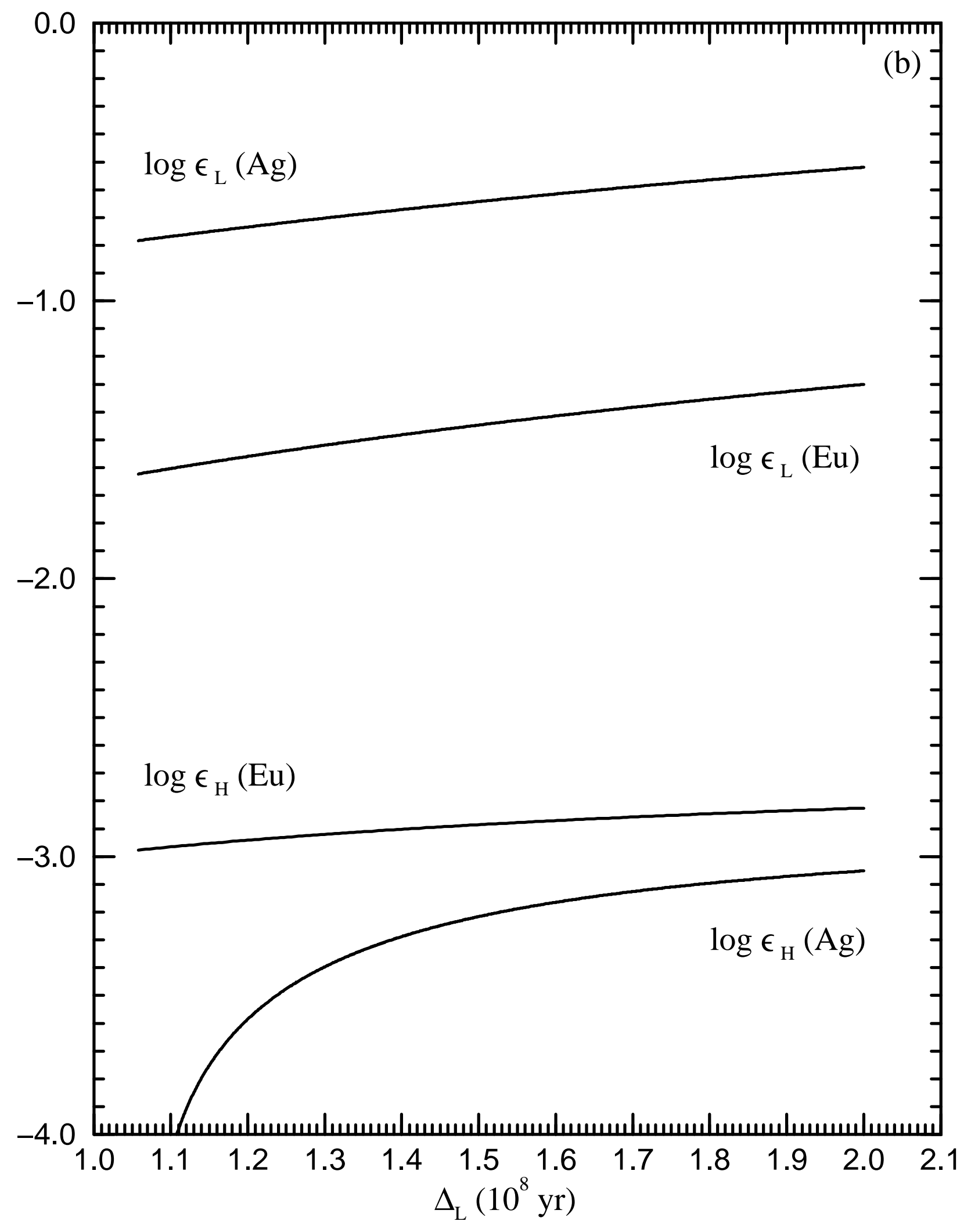




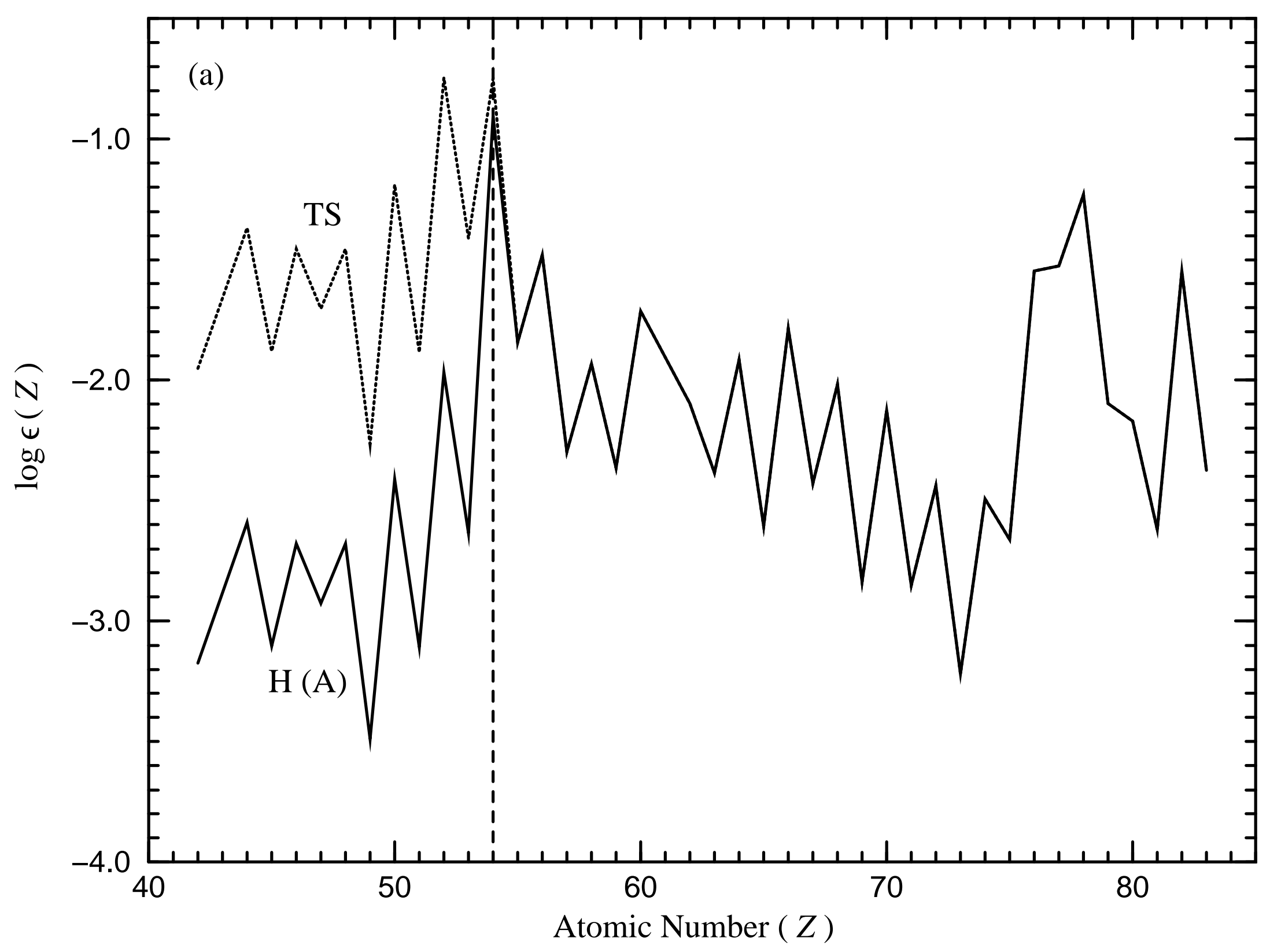




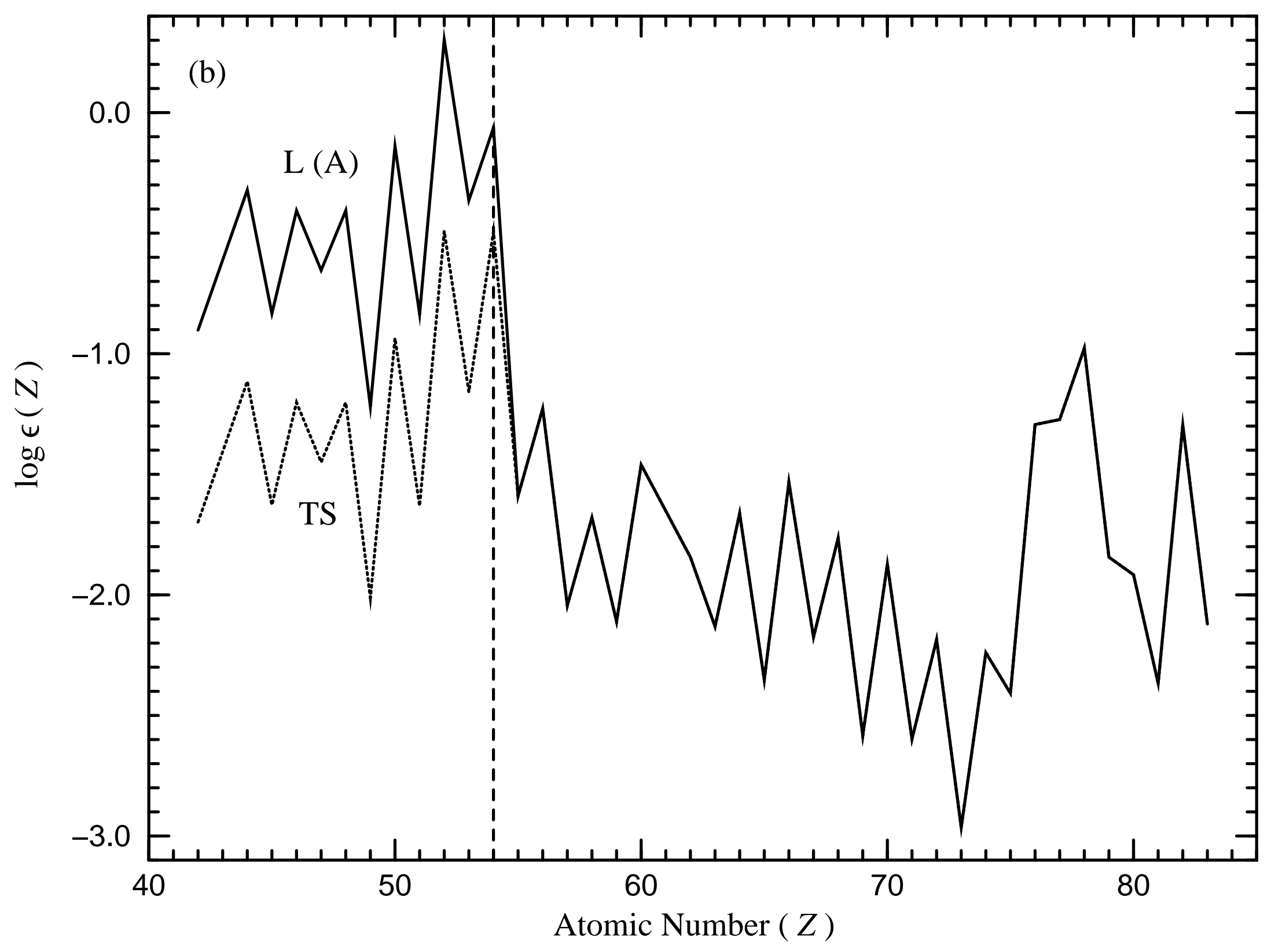









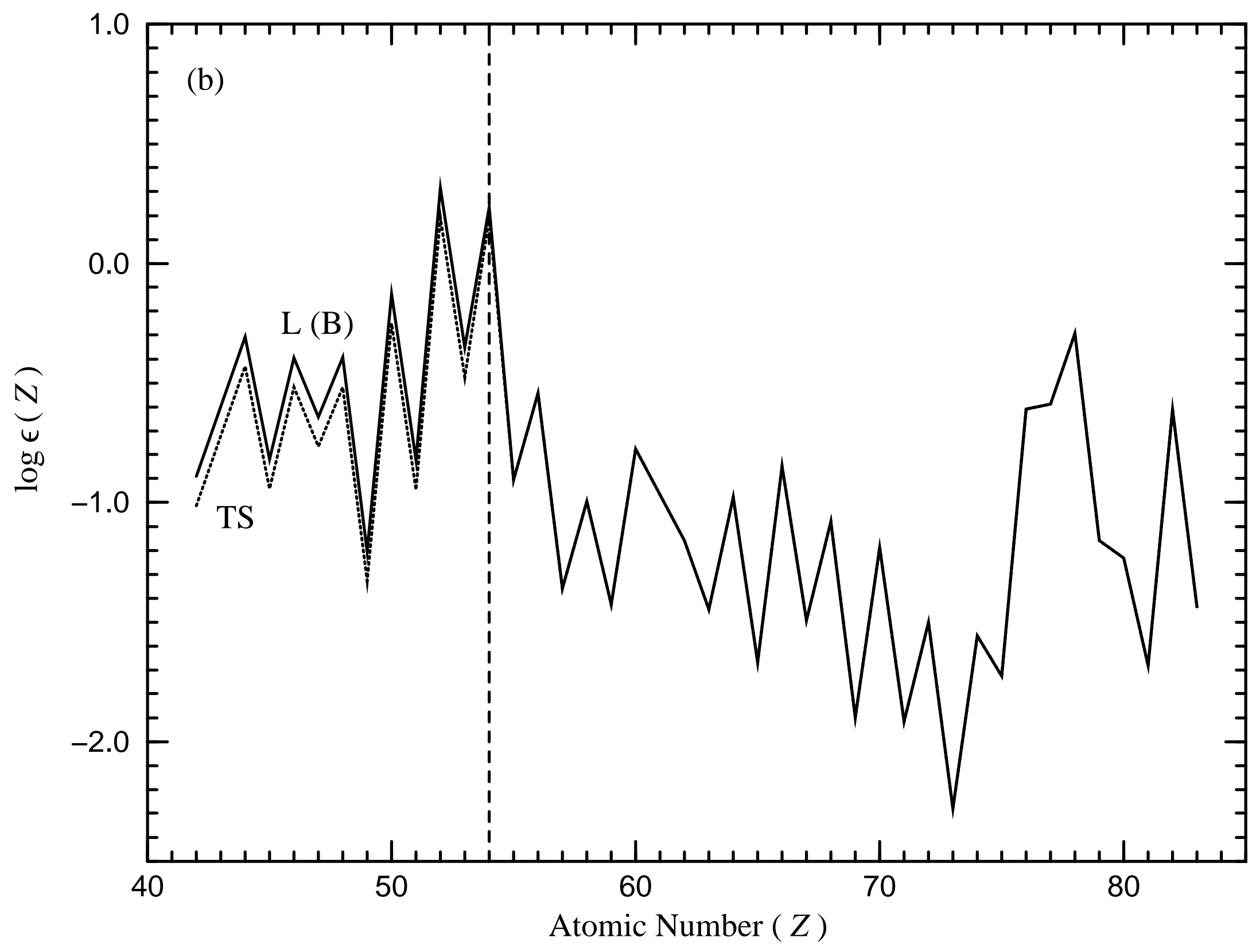




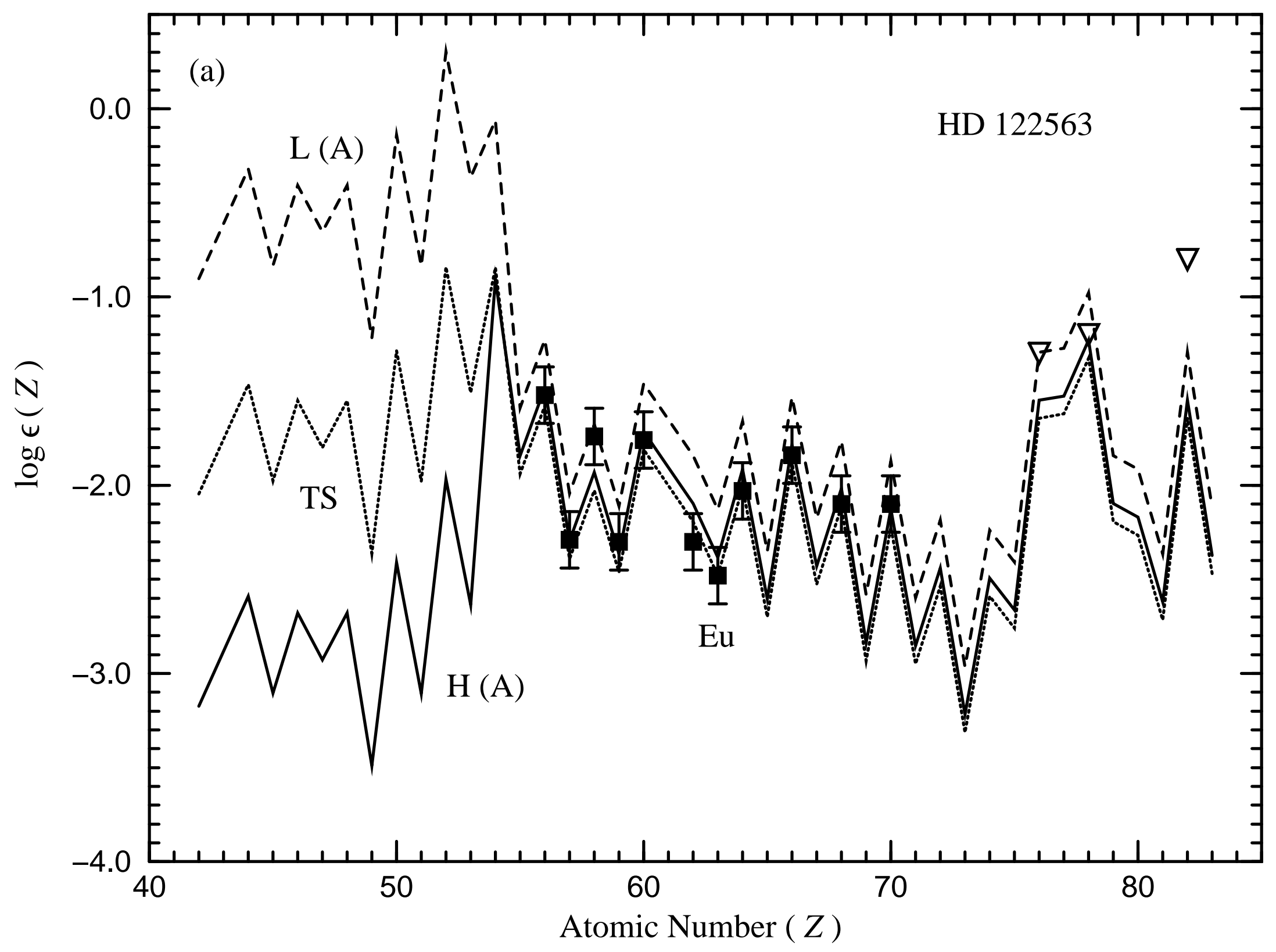




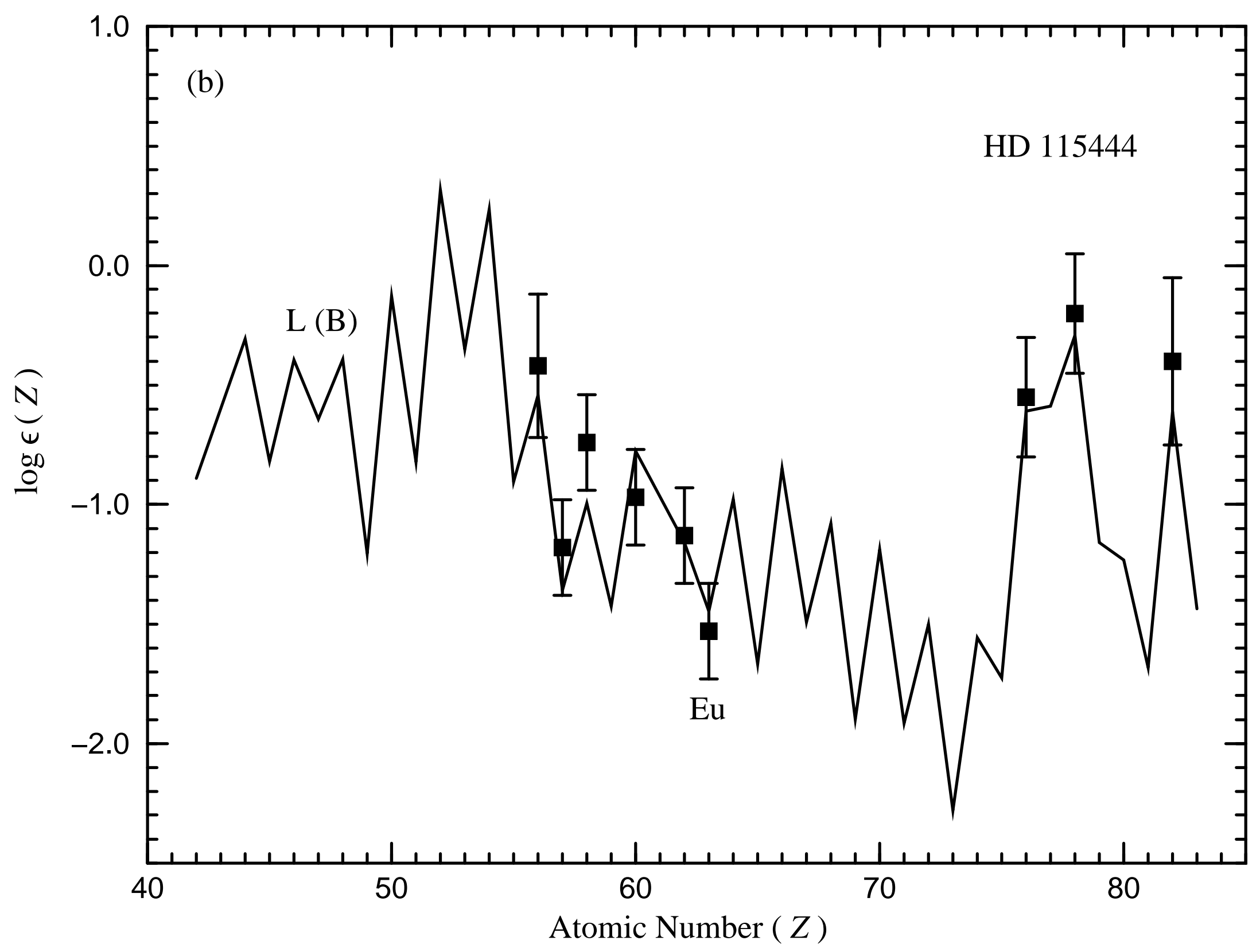




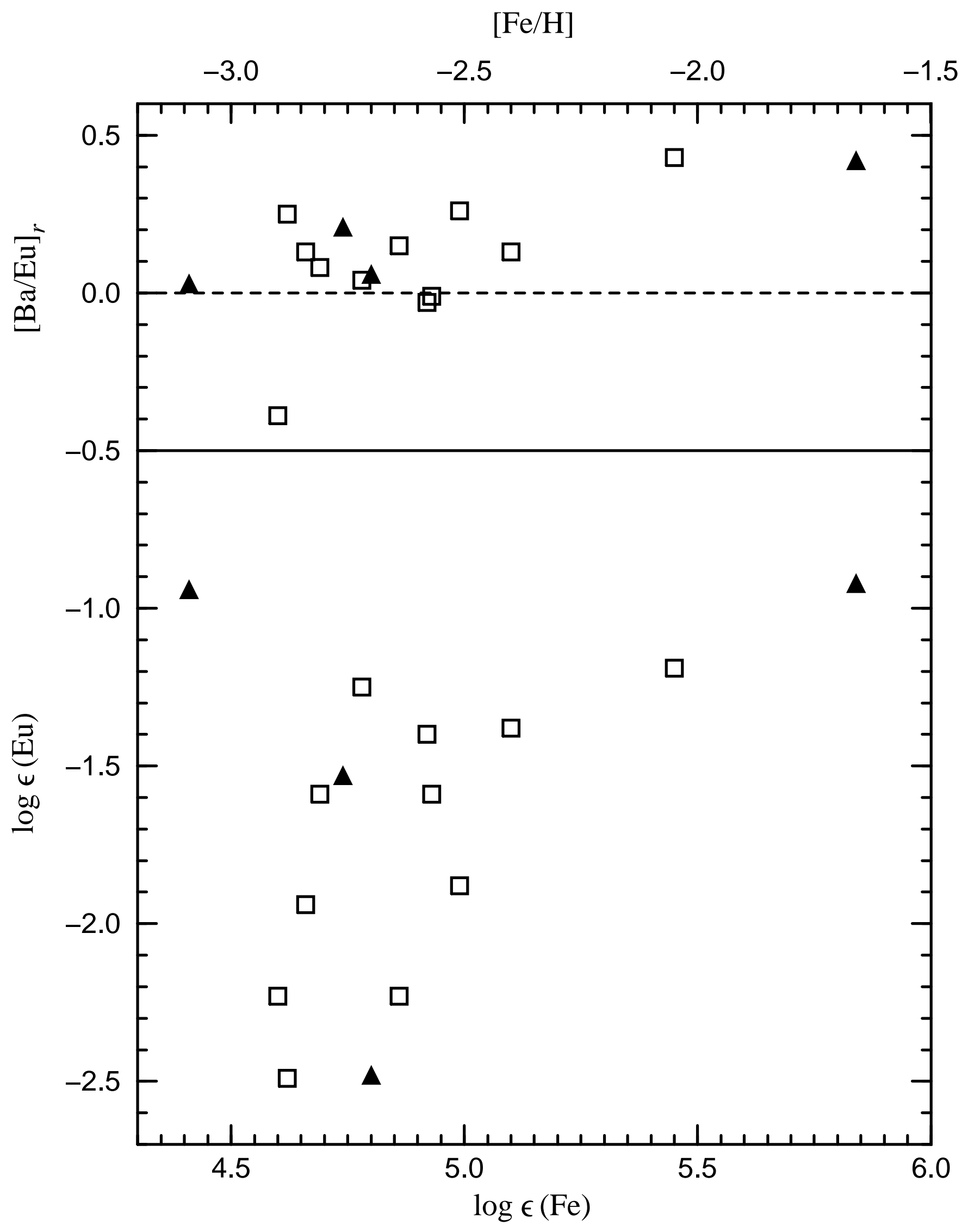




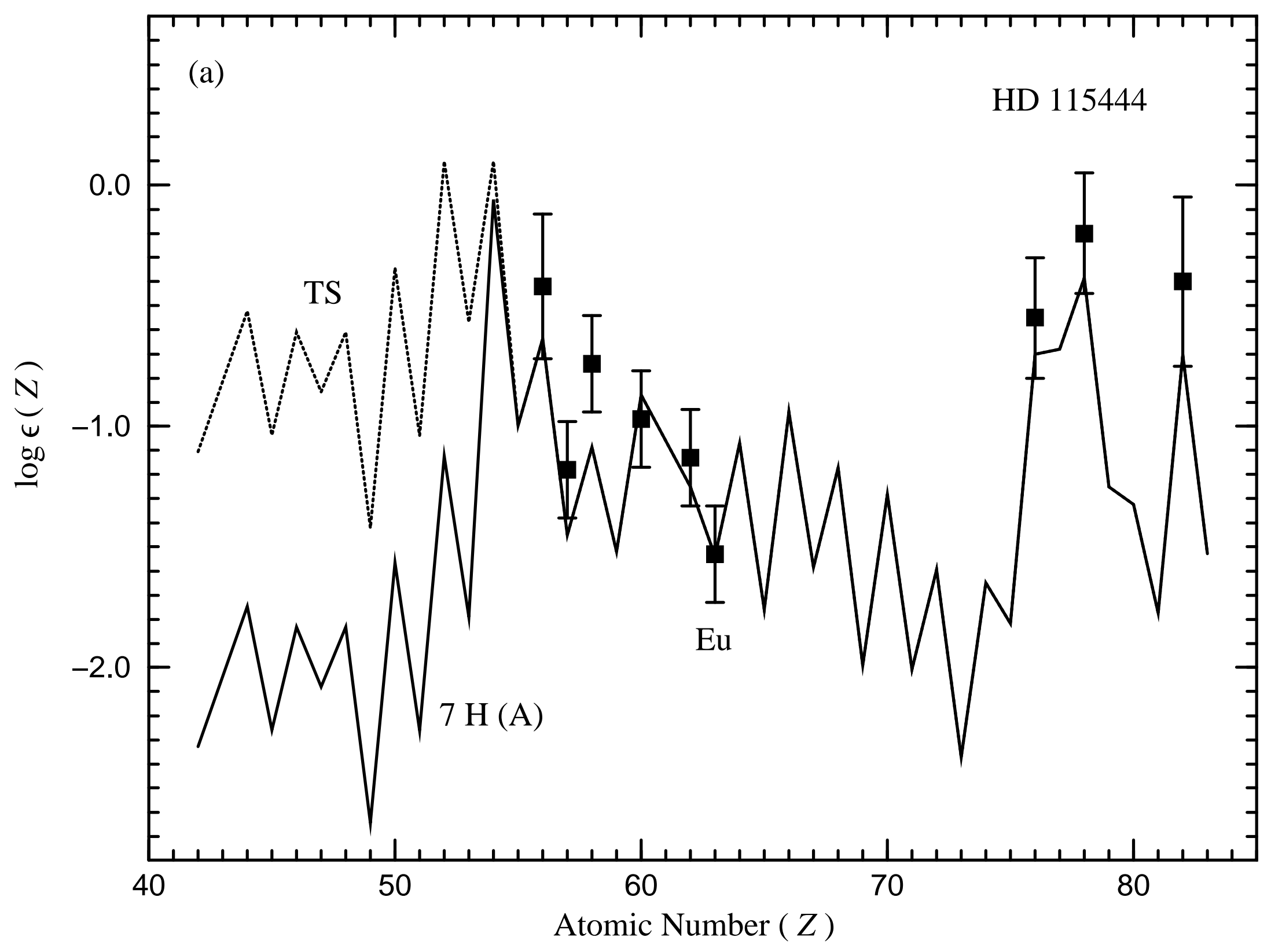




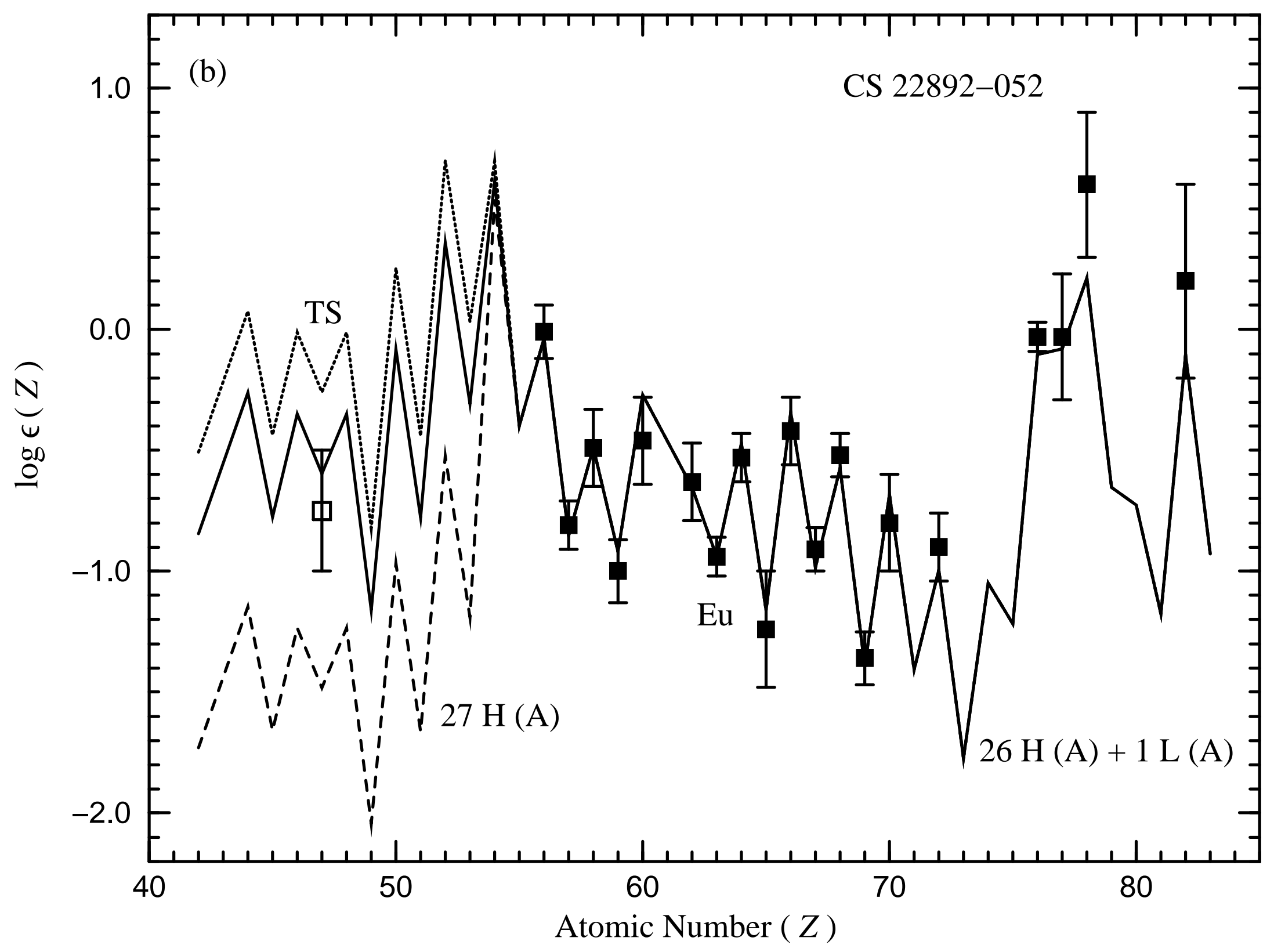

